\title{
FLEET Velocimetry Measurements on a Transonic Airfoil
}

\author{
Ross A. Burns ${ }^{1}$ and Paul M. Danehy ${ }^{2}$ \\ NASA Langley Research Center, Hampton, VA, 23681
}

\begin{abstract}
Femtosecond laser electronic excitation tagging (FLEET) velocimetry was used to study the flowfield around a symmetric, transonic airfoil in the NASA Langley $0.3-\mathrm{m}$ TCT facility. A nominal Mach number of 0.85 was investigated with a total pressure of $125 \mathrm{kPa}$ and total temperature of $280 \mathrm{~K}$. Two-components of velocity were measured along vertical profiles at different locations above, below, and aft of the airfoil at angles of attack of $0^{\circ}, 3.5^{\circ}$, and $7^{\circ}$. Measurements were assessed for their accuracy, precision, dynamic range, spatial resolution, and overall measurement uncertainty in the context of the applied flowfield. Measurement precisions as low as $1 \mathrm{~m} / \mathrm{s}$ were observed, while overall uncertainties ranged from 4 to 5 percent. Velocity profiles within the wake showed sufficient accuracy, precision, and sensitivity to resolve both the mean and fluctuating velocities and general flow physics such as shear layer growth. Evidence of flow separation is found at high angles of attack.
\end{abstract}

\section{Nomenclature}

General Symbols

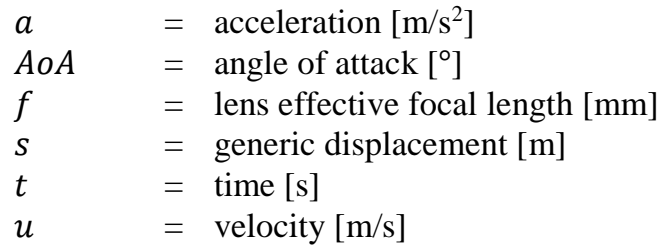

Greek Symbols

$\alpha=$ angle between $x$ - and $z$ - components of velocity [ $\left.{ }^{\circ}\right]$

$\gamma=$ angle between $x$ - and $y$ - components of velocity $\left[{ }^{\circ}\right]$

$\delta \quad=$ generic uncertainty

$\varepsilon \quad=$ generic error

$\sigma \quad=$ generic standard deviation or precision

$\psi \quad=$ angle of rotation about tunnel $z$-axis $\left[{ }^{\circ}\right]$

$\theta \quad=$ angle between tunnel $x$-axis and camera viewing axis $\left[{ }^{\circ}\right]$

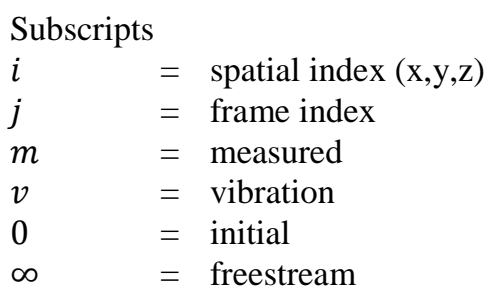

\section{Introduction}

$\mathrm{T}$ RANSONIC cryogenic wind tunnels produce flight-accurate Reynolds numbers in ground-testing environments. Facilities such as the European Transonic Windtunnel (ETW) and the National Transonic Facility (NTF) at NASA Langley Research Center regularly see testing from commercial and research partners alike for this reason. Transonic

${ }^{1}$ Research Engineer, National Institute of Aerospace, AIAA Member.

${ }^{2}$ Research Scientist, Advanced Measurements and Data Systems Branch, AIAA Associate Fellow.

American Institute of Aeronautics and Astronautics 
cryogenic tunnels (TCTs for brevity) achieve these high-Re operating conditions by injecting liquid nitrogen into the facility flow circuits, which (upon evaporation) reduces the operating temperature and simultaneously increases the flow density and decreases the viscosity. ${ }^{1,2,3}$ While this mode of operating is advantageous for simulating aerodynamic effects, it necessitates a rugged construction to accommodate the high pressures and thermal stresses present in and around the flow path. Lack of optical access is common in TCT facilities, limiting the variety of diagnostic techniques that can be used. Data for customer tests in this type of facility are thus commonly limited to integrated force and moment measurements and more traditional probe-based pressure and velocity measurements. ${ }^{4}$ However, since probebased measurements intrinsically disturb the flowfield of interest, there is a need for less intrusive diagnostics for the measurement of relevant flow quantities. ${ }^{5}$ For example, a great deal of work has gone into the development of pressureand temperature-sensitive paints that operate in cryogenic, oxygen-free environments for measuring surface conditions and detecting turbulent boundary layer transition on test articles. ${ }^{5,6,7}$ However, measurements of velocity have proven very challenging to researchers. Techniques such as particle image velocimetry ${ }^{9}$ and Doppler global velocimetry ${ }^{10}$ have been applied in ETW and its pilot facility; seeding was introduced by injecting steam-saturated nitrogen into the flow circuits and allowing the water vapor to condense at decreased temperatures. However, this and other artificial seeding methods are prohibited in most TCT facilities, including those at NASA Langley Research Center, for fear of contaminating the flow circuits with particulates or residual water vapor or oils, which can condense on test models causing icing or surface roughness. Consequently, the measurement of velocities through optical diagnostic techniques has been limited at TCT facilities. At NASA Langley, only two different optical velocimetry techniques have been applied in their TCT facilities (prior to the present body of work of the authors), both occurring in the 0.3-m Transonic Cryogenic Tunnel. One study by Gartrell et al. utilized laser Doppler anemometry to study the freestream velocity and fluctuations, ${ }^{11}$ while the other study by Honaker and Lawing used a laser transit anemometer to study the flowfield near a cylinder. ${ }^{12}$

The present studies focus on a technique called femtosecond laser electronic excitation tagging (FLEET) velocimetry, ${ }^{13}$ in which the luminescence from a plasma generated by focusing a femtosecond laser pulse is tracked through sequential imaging to measure velocities. FLEET is part of broader class of techniques which currently includes PLEET ${ }^{14}$ (picosecond laser electronic excitation tagging) and STARFLEET ${ }^{15}$ (selective two-photon absorptive resonance FLEET). All of these techniques have their niche applications. FLEET is simplest of these techniques and requires the least specialized equipment (single camera with an intensifier and a single laser), but the large thermal perturbation ${ }^{15,16}$ resulting from the excitation process could potentially cause velocity lag in the tagged volume and alter flowfields of interest. STARFLEET bypasses this issue by using a tuned laser source to excite nitrogen, reducing the overall amount of energy required by nearly two orders of magnitude. However, the deep ultraviolet wavelengths at which the excitation is optimized make long-distance transmission of the beam difficult, requires special windows and optics for the facilities in which it is used, and also requires additional harmonic generation optics for femtosecond laser systems. PLEET utilizes a pulse with a much longer duration with higher energy content to achieve a similar excitation of nitrogen as FLEET. PLEET can also be adapted to operate at very high repetition rates (up to $100 \mathrm{kHz}$ ), making time-resolved, unseeded velocity measurements possible. Due to the high pulse energies required to generate the PLEET effect, the temperature rise is more substantial than that of FLEET.

Since the FLEET technique requires no seeding beyond nitrogen (which is present in abundance in most facilities) and is experimentally simple to implement, it is being investigated for its use as a velocity measurement technique that could be applied in TCT facilities such as the NTF. FLEET velocimetry has been previously demonstrated in the NASA Langley $0.3-\mathrm{m}$ TCT facility as an excellent marker for velocity measurements in the tunnel freestream ${ }^{17}$ and has the potential to measure thermodynamic properties as well. ${ }^{18}$ The present study seeks to examine and document the utility of FLEET for making measurements in much more complex flow geometries, specifically those around models. To this end, the flow around a transonic airfoil model in the $0.3-\mathrm{m}$ TCT facility is used as a test bed for making FLEET velocity measurements in such an environment. A discussion and analysis of the measurement accuracy, precision, dynamic range, spatial resolution, and overall measurement uncertainty are given in the context of this applied measurement. Special issues related to applied flows are also examined. Finally, the flowfields around the transonic airfoil at varying angles of attack $(A o A s)$ are examined. These measurements serve to legitimize the FLEET velocimetry technique for making applied measurements in large-scale and TCT facilities.

\section{Experimental Program}

\section{A. Test Facility}

All tests were conducted in the NASA Langley 0.3-m Transonic Cryogenic Tunnel (0.3-m TCT); a closed-loop, fan-driven wind tunnel capable of operating a Mach numbers ranging from 0.2 to 0.9 . While able to operate on a number of test gases, all tests were conducted in nitrogen $\left(\mathrm{N}_{2}\right)$. Operating total pressures ranged from 100 to $500 \mathrm{kPa}$,

American Institute of Aeronautics and Astronautics 
but were kept constant at $125 \mathrm{kPa}$ during these studies. The total temperature during testing was held at $280 \mathrm{~K}$. The Reynolds number based on the airfoil midspan chord length ranged from 0.5 to 1.6 million. The facility is constructed with a doubleshelled design; the central test section (or test cell) is surrounded by a pressurized plenum of nominally quiescent gas. This construction allowed the presence of either adjustable or slotted walls in the test section. The test section has cross-section dimensions of approximately $0.33 \mathrm{~m} \times 0.33 \mathrm{~m}$. Unlike previous tests by the authors, ${ }^{17,18 \text { Error! Bookmark not defined., } 19}$ the test section was set to have slightly diverging walls to accommodate the additional blockage imposed by the test model.

Optical access to the facility was afforded by three fused silica windows penetrating the plenum and test section. A single large, circular window penetrated the outer plenum wall, while a pair of windows was situated in the wall of the inner test section to allow optical access to the inner test cell. This window configuration can be seen in Fig. 1.

\section{B. Airfoil model}

The present study utilized a symmetric, semi-span airfoil model. This model was tapered in both the transverse and span-wise direction. Cross-sectional profiles of the airfoil can be seen in Fig. 2. The total span-wise extent of the model was $150 \mathrm{~mm}$. The airfoil model was mounted from a rotatable turntable situated inside one of the tunnel sidewalls to allow for the angle of attack to be maintained and adjusted by the facility control systems. The center of rotation was located at the trailing edge of the airfoil at approximately the mid-span location. A perspective view of the airfoil model and turntable relative to the optical access is shown in Fig. 1. Note also the coordinate system used in these studies, referenced in Fig. 2. The stream-wise coordinate corresponds to the $x$-direction and has its origin at the leading edge of the base of the airfoil when at an $A o A$ of $0^{\circ}$. The transverse coordinate is aligned with the $y$ direction and has its origin at the same location.

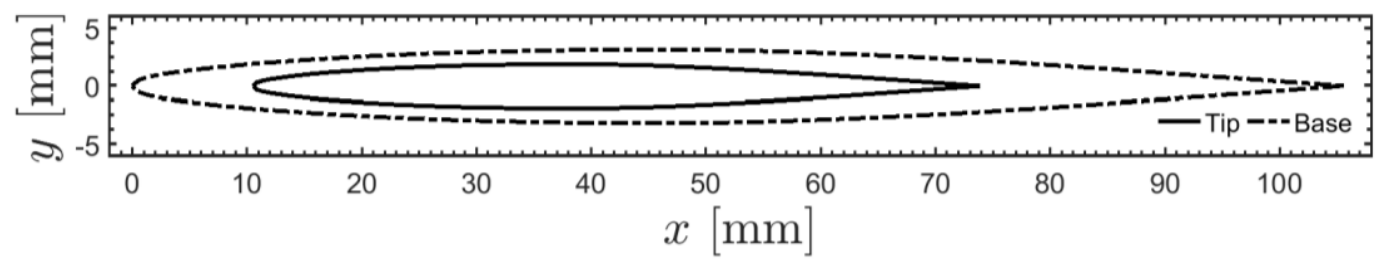

Figure 2. Airfoil cross-sectional profiles.

\section{Optical systems}

These tests utilized a regeneratively-amplified Ti:Sapphire laser system (Spectra-Physics Solstice) with a repetition rate of $1 \mathrm{kHz}$, center wavelength of $800 \mathrm{~nm}$, and a bandwidth of $20 \mathrm{~nm}$. An approximate energy of $1 \mathrm{~mJ} / \mathrm{pulse}$ was used in these measurements, though up to 50 percent of this energy was attenuated through the various optical elements in the setup. The output from the laser system, which was located approximately $3 \mathrm{~m}$ above the test section, was routed through a system of periscopes to the height of the facility and through the outer plenum window. Inside the plenum, the beam was steered through remotely adjustable horizontal and vertical periscopes to allow the position of the beam with respect to the model to be changed. The laser was then directed into the test section and focused through a pair of closely spaced lenses in series $(f=+500 \mathrm{~mm}$ and $f=+1000 \mathrm{~mm}$, effective focal length $330 \mathrm{~mm})$. This optical arrangement placed the FLEET signal approximately $100 \mathrm{~mm}$ from the base of the airfoil and far wall of the test section. A diagram of the optics in and around the facility are shown in Fig. 3. Vertical velocity profiles were captured at two stream-wise locations downstream of the airfoil (located at $88.4 \mathrm{~mm}$ and $102.9 \mathrm{~mm}$ by the coordinate system in Fig. 2) and at five stream-wise locations on the top surface of the airfoil (31.25 mm, $40 \mathrm{~mm}, 48.75 \mathrm{~mm}$, $57.5 \mathrm{~mm}$, and $66.25 \mathrm{~mm}$ ). To make measurements on both the compression and expansion surfaces of the airfoil, these 
same measurement locations were used at both positive and negative $A o A s$ (e.g. $\pm 7^{\circ}$ ). Although measurements could be made as close as $1 \mathrm{~mm}$ from the surface of the model, often a larger offset was used to prevent any possible damage to the model.

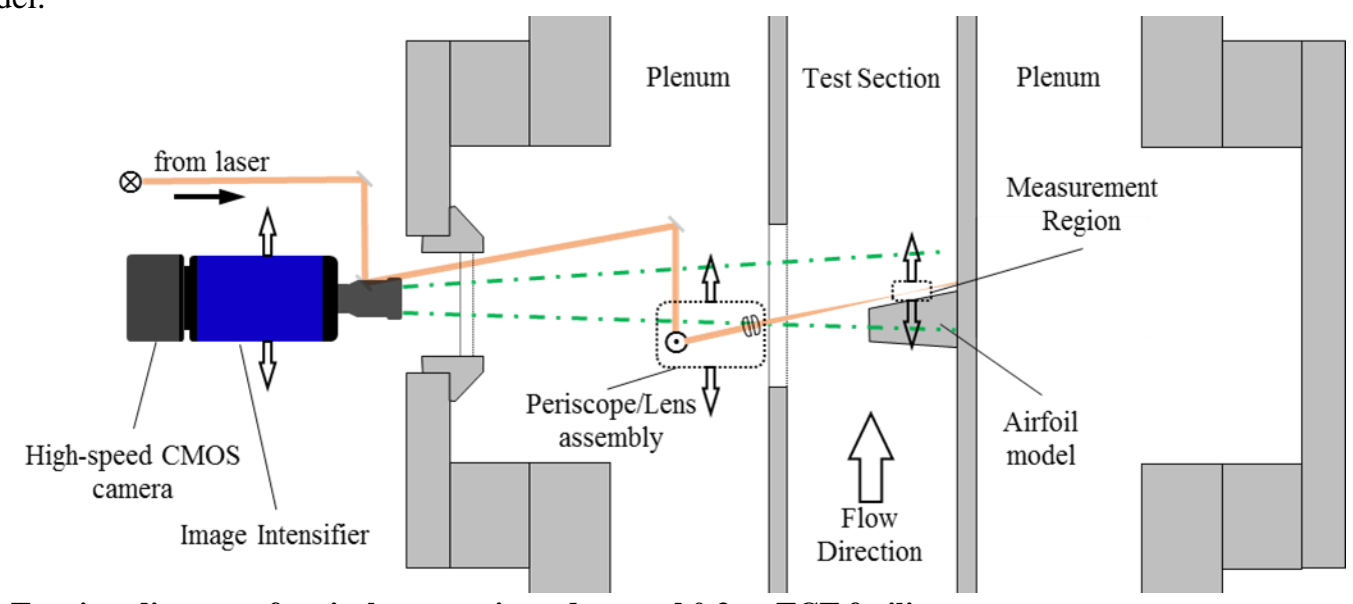

Figure 3. Topview diagram of optical systems in and around 0.3-m TCT facility.

\section{Data acquisition systems}

Imaging of the FLEET signal was done using a high-speed image intensifier (LaVision HS-IRO) lens-coupled to a CMOS camera (Photron Mini AX-200). The camera system was operated in a triggered burst-mode; the camera's overall acquisition rate was $100 \mathrm{kHz}$, and 100 bursts of 6 frames were captured every second. This timing configuration was used to increase the testing duration, ensuring that several full tunnel transit times occurred while the laser was at each sampling location. The intensifier was triggered variably during these bursts; two gates occurred at the end of the third frame and beginning of the fourth frame with a temporal separation of $4 \mu$ s, while two subsequent gates occurred at the beginning of each of the last two camera exposures in each burst. A timing diagram is shown in Fig. 4. This arrangement was used to ensure at least two viable frames of data were captured in the event of a short signal lifetime while still providing ample temporal information about the propagation of the signal. Note also that the two frames captured prior to the laser pulse are used in the post-processing of the data (the first frame being contaminated by excessive thermal noise is discarded and the second frame provides a background image). As in previous tests, the FLEET signals were viewed in a quasi-boresight configuration; the camera line-of-sight was nearly parallel (approximately $14^{\circ}$ off-axis) with the direction of beam propagation. This approach was used so that images could be acquired from the same window where the laser is directed into the flow. Unlike previous tests, the entire camera/intensifier system was mounted to a translation stage so that it could be moved with the laser.

The 0.3-m TCT facility had an extensive data acquisition system (DAS), consisting of an array of wall pressure taps, thermocouples, pitot probes, pressure transducers, and strain gauges to measure relevant properties of the flow. These probes were situated throughout the test section walls and within the plenum. Data from this equipment was read into facility computer systems for processing. A facility data point was collected during each FLEET data acquisition run to measure the facility conditions corresponding to the FLEET test point.

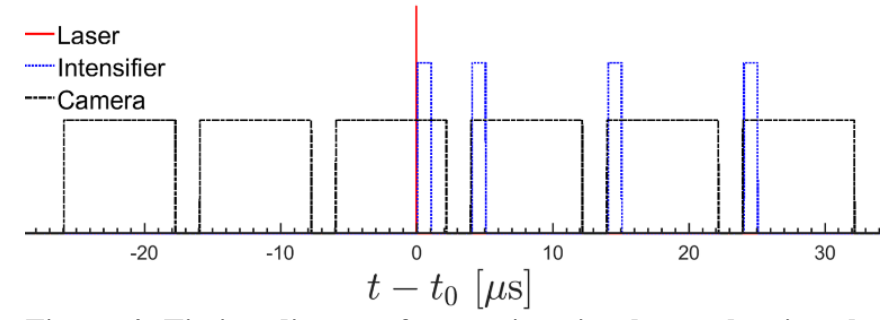

Figure 4. Timing diagram for one imaging burst showing the relative delays between the camera exposures, laser, and intensifier gates.

\section{Data Analysis}

The raw FLEET image data were subjected to multiple stages of pre- and post-processing to extract velocity information. For reference, a sample burst of images is shown in Fig. 5, which was a data sample taken in the wake of the airfoil model operating at Mach 0.85 , total temperature of $280 \mathrm{~K}$, and total pressure of $125 \mathrm{kPa}$. 


\section{A. Displacement calculations}

Unlike the previous experiments done in this facility using FLEET, displacements were measured from the raw images through a custom adaptive-crosscorrelation (ax-corr) routine. This scheme was used because it allowed for comparatively rapid processing of the data than the hybrid surface-fitting/crosscorrelation scheme used previously, while maintaining the same measurement precision. No intensity information can be directly extracted through this process, nor are absolute positions derived, both of which are handled in a separate step (described in the Section III.B). The basic kernel of the adaptivecross-correlation routine operated by first identifying a reference subimage within one of the frames of data that contained all of the FLEET signal in that particular image. Typically this region was around 15 $p x \times 20 p x$, though its size varied from image to image as the size and shape of the FLEET signal changed. A second region of interest was determined in the raw FLEET data, which would contain all the signal occurring within a particular burst of images. This second subregion was used to increase the speed of processing, which scaled directly with the size of the image. Next, two-dimensional cross-correlation was performed between the reference subimage and the second region of interest in each frame of data. Displacements with subpixel accuracy were then determined from the resulting correlation maps by fitting a two-dimensional Gaussian function in an $11 \mathrm{px} \times 11 \mathrm{px}$ region around the principal correlation peak (which was significantly higher in magnitude than the surrounding correlation noise). The standard $\mathrm{R}^{2}$, signal-to-residual ratio (SRR), and ratio of the primary correlation peak to the second strongest correlation peak were recorded for each correlation map. These displacements were then referenced to the first frame of data; the displacements were offset such that the displacement in the first frame of data was zero by definition. This processing kernel was repeated four times on each burst of data, changing which of the four frames of data was used as the reference. Thus, each of the three displacements from the four frames of data is measured four times. The displacements used in all subsequent calculations were the average of these four separate calculations (or all valid samples). Rejection criterion used were an $\mathrm{R}^{2}$ value below 0.95 , signal-to-residual ratio below 100 , or a correlation peak ratio below 3 . Typically the $\mathrm{R}^{2}$ criterion was the most restrictive.

\section{B. Surface fitting algorithm}

Independent of the displacement calculations, the FLEET data were analyzed to extract information about absolute position and intensity. Specifically, the first frame of data in each burst was run through the SRGE (shifted-rotated generic ellipsoid) surface-fitting algorithm, which is described in Ref. 18. The SRGE algorithm yields the FLEET spot peak intensity, integrated intensity, centroid location, and location of peak intensity in image coordinates. Using these position data in conjunction with the displacement data calculated with the ax-corr algorithm, the absolute position of the FLEET signal could be determined for each frame. These data are used in the calculation of velocities that follows.

\section{Spatial calibration}

A brief note about calibration is given here to distinguish the procedure from previous tests. The usual procedure during testing utilized a single fixed-position target pattern (dotcard) that was inserted into the focus of the camera after properly focusing on the FLEET signal. Note that because of the high intensity off the fs laser and the boresight imaging configuration, the target pattern could not be placed or imaged while the laser was active. An image of this target pattern was then run through a custom de-warping and scaling algorithm to correct the images and bring them into a physical coordinate system. However, in the post-processing of the velocity data from the previous testing campaign, it was established that due to the large depth of field, there was considerable uncertainty in the placement of the target in this fashion. Consequently, a new procedure was adopted to eliminate bias in the velocity measurement; the target was scanned through the entire depth-of-field (approximately $1.5 \mathrm{in}$ ), and the pattern size and degree-offocus were evaluated to determine when the position was optimal. Specifically, the calibration image with the highest

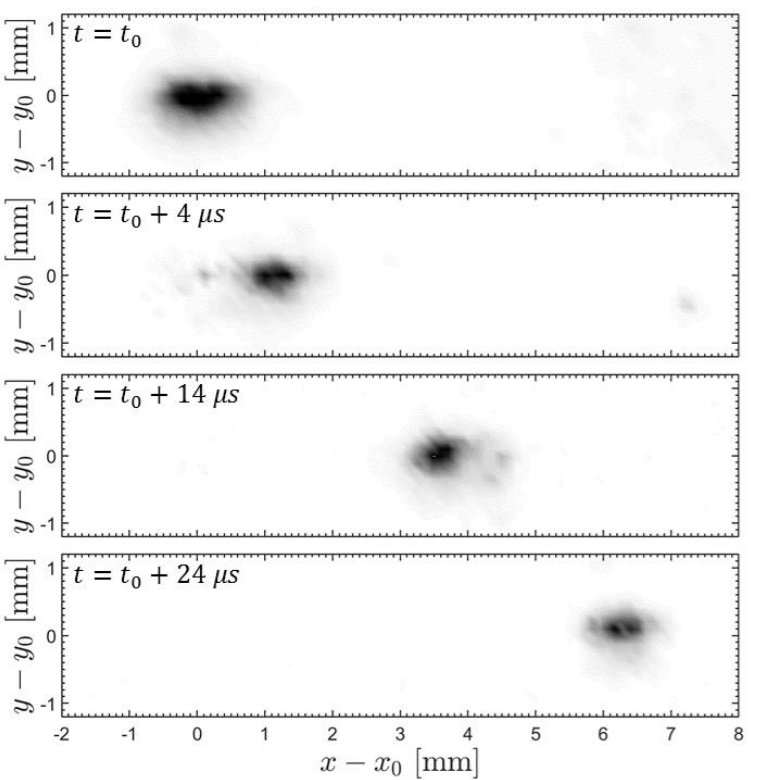

Figure 5. Sample sequence of FLEET images. Data was taken at Mach 0.85, $P_{t}=125 \mathrm{kPa}$, and $T_{t}=280 \mathrm{~K}$. 
degree-of-focus (assumed to be the image with the smallest diameter spots in the target) was used in the calibration procedure. The scanning procedure also helped to place uncertainty bounds on the velocimetry measurements, which will be discussed further in the Section IV and Appendix A.

\section{Velocity calculations}

A variety of different models were used to convert the measured displacements into velocities. These methods include the point-to-point method, a linear regression method, and a polynomial fitting method. In the actual velocity data, the optimal velocity was selected based on criteria that will be discussed below.

\section{Point-to-point method}

The point-to-point method is the standard time-of-flight velocimetry calculation typical of molecular-tagging velocimetry techniques. In this method, the velocity at a point is calculated by making a finite-difference approximation of the displacement derivative:

$$
u_{i, j}=\frac{\Delta s_{i, j}}{\Delta t_{i, j}}
$$

where $u$ is the velocity, $\Delta s$ is the displacement, and $\Delta t$ is the temporal separation. The indices $i$ and $j$ refer to the vector direction $(\mathrm{x}, \mathrm{y}, \mathrm{z})$ and frame number, respectively. Note that since variable timing was used in each burst, the $\Delta t$ term varies between frames. In this manner, three velocities at different starting positions can be evaluated for each burst of data.

\section{Linear regression method}

Linear regression allows all measured locations within a given trajectory to be consolidated in a single velocity calculation. By assuming a functional form for the displacement to be that of a linear function, the velocity evaluation can be simplified greatly:

$$
s_{i, 1 \rightarrow j}(t)=u_{i, j} t+s_{0}
$$

Here the subscript $1 \rightarrow j$ indicates that the displacement corresponds to all images from frame 1 to frame $j$ within a given burst. Two such velocity calculations are conducted for each burst: all points from frames 1 to 3 and again with all frames from frames 1 through 4 . The initial position in the trajectory was set to zero to further accommodate this fitting procedure. The velocity, $u$, was evaluated using a least-squares fitting algorithm, and the $\mathrm{R}^{2}$ value was calculated for each velocity.

\section{Polynomial fitting method}

Much like the linear regression model discussed in the Section III.D.2, the polynomial fitting method uses a higherorder equation for fitting the FLEET signal trajectory. In this case the trajectory was limited to a quadratic function:

$$
s_{i, 1 \rightarrow j}=\frac{1}{2} a_{i, j} t^{2}+u_{0, i, j} t+s_{0}
$$

The additional term $a$ is a nominal acceleration through the trajectory. This fitting method was utilized only when four data points were available in a given burst, and thus only one such calculation was made. Again, the initial position was subtracted from the individual positions, and thus the $s_{0}$ term was often negligible. As with the linear regression model, the $\mathrm{R}^{2}$ value was calculated for each fit made. The velocity used in calculations was either the initial velocity prescribed by the fit parameter $u_{0, i, j}$ or the mean velocity prescribed by Eq. (3) over the time interval of the measurement.

\section{Optimal velocity}

The different velocity evaluation methods were used because they have been shown through the previous FLEET work to yield varying levels of accuracy and precision, the linear regression method exhibiting the highest of all of these methods. ${ }^{18}$ However, the trajectories experienced in the airfoil flowfield are much more varied in form (streamline curvature and a higher degree of unsteadiness) than in the relative calm of the freestream that had been evaluated previously. Thus, it was necessary to accommodate numerous possibilities for the trajectory so that the behavior of the underlying flow could be accurately represented. Thus, a combination of these different evaluation methods was often employed within a given data set depending on what the flow trajectories were. Specific criteria were put in place to determine which of these calculated velocities were to be used. First, a threshold $\mathrm{R}^{2}$ value of 0.97 was used to filter the two regression-based models in quality. This filter was applied to both components of velocity, and if one component did not pass the filter, both components were rejected. If all velocities passed this simple check, the velocities were prioritized as follows for each sample: 1) linear regression with four data points, 2) polynomial 
regression, 3) linear regression with three data points, and 4) point-to-point. The logic behind this selection was evidentiary. Typical pathlines in the freestream of the facility were very straight, and could be well-represented by the linear regression model. However, the airfoil flowfield often exhibited subtle streamline curvature, which could be accurately represented by the polynomial fitting method. The high threshold on the fit $\mathrm{R}^{2}$ value served as a discriminating factor between these two behaviors, and was often most visible in the $y$-component of velocity. The third selection criterion was used when the data could still be evaluated at three points, but the fourth point was invalid for any number of reasons (low SNR and poor correlation quality, typically). Finally, the point-to-point method was used when the streamline curvature became very strong, such as in what was found to be a separated flow region in the wake of the airfoil. When this was the case, often the two regression-based models had very poor fit quality, and the point-to-point evaluation method was used to establish the velocity. As will be discussed in some detail in Section IV, these same regions also suffered from a reduced signal lifetime, making the other evaluation methods difficult to implement.

\section{E. Velocity profile sampling}

To construct velocity profiles, notional data sampling regions were placed around the initial locations of the FLEET signal encountered during a single traverse of the scanning system. The average velocity and displacement for all FLEET spots falling within these regions were then included in the associated statistics. The sampling regions were given a transverse extent of the $0.7 \mathrm{~mm}$, corresponding to the mean full-width, half-maximum (FWHM) FLEET spot diameter (and thus a limit on the spatial resolution). The sample regions were overlapped by 75 percent. A more detailed discussion of spatial resolution as it pertains to these measurements is given in Section IV.

\section{Results and Discussion}

The conditions under which FLEET is being applied in these studies are significantly different in character to those studied by the authors to date, which have been relatively 'well-behaved' freestream flow environments. In particular, the flow over the transonic airfoil is subject to a wide range of velocities spanning from supersonic regions (up to nearly Mach 2) to zero- and negative-velocity regions. Turbulent, recirculating, transient, and otherwise unsteady flow features are prominent in certain circumstances, such as having the airfoil at a high $A o A$ and high Mach number. Furthermore, there are large mean velocity gradients in the flowfields as well as significant streamline curvature. Accompanying many of these different effects are large spatial variations in thermodynamic properties as well. Finally, the presence of the model also imposes a limitation on where measurements can be made. After discussing these issues broadly, a specific airfoil flowfield is examined and interpreted in the context of the measurement capabilities.

\section{A. Measurement Assessment}

\section{Dependence of FLEET signal lifetime and intensity on strain rate}

While this observation is a fairly specific point to bring up first, it informs many of the subsequent points and thus warrants discussion at the onset. FLEET is fundamentally different than many other molecular tagging velocimetry (MTV) techniques in that it doesn't utilize repeated excitation of a particular tagged species. Rather, the excitation process generates a volume of gas with self-sustaining fluorescence that gradually depletes itself over tens of $\mu \mathrm{s}$. If left otherwise undisturbed, the lifetime of the signal appears to be a strong function of the local density of the gas in which it was created, and in fact has been used to measure thermodynamic properties of nitrogen using this property. ${ }^{18}$ However, during a previous study it was observed that the lifetime of the FLEET signal appeared to be dependent on the unsteadiness of the gas. That is, in regions where there appeared to be a higher degree of unsteadiness, turbulence, or other rapid transient flow behaviors, the FLEET signal lifetime decreased substantially. The FLEET spot or line appears to "break up," and fluorescence could only be seen for a short time. No quantification of this observation was made, but it is a very important result in the context of an

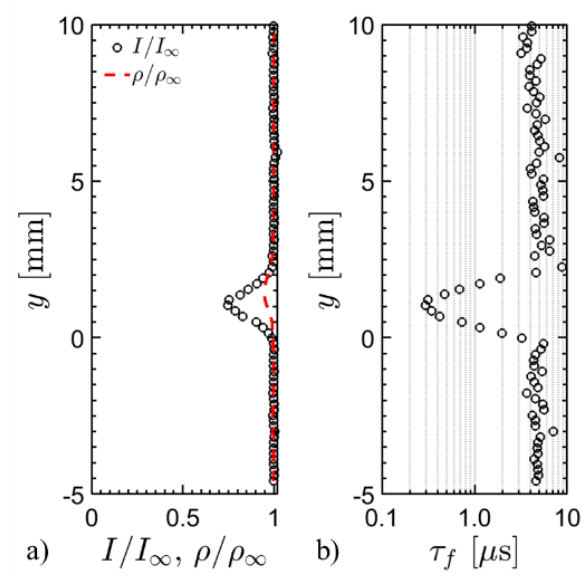

Figure 6. FLEET signal variation in Mach 0.85 wake flow. a) Measured signal intensity and estimated density and b) measured signal lifetime.

American Institute of Aeronautics and Astronautics 
applied measurement such as these studies. A shorter signal lifetime results in lower observed signal intensities and fewer frames of data to consider in the velocity calculations, both of which adversely affect the precision of the velocity measurements. Furthermore, the potential for using the FLEET signal intensity as a density indicator (as discussed in Ref. 18) is dependent on the signal lifetime to an extent. Thus it is important to understand the magnitude of this effect in the context of the applied measurement. Figure 6a shows a measured intensity profile in one Mach 0.85 wake flow. It can be seen that the signal is uniform throughout the regions flanking the central velocity deficit. Since there isn't expected to be a significant variation in density, the signal intensity should remain roughly constant throughout these regions. In the studies in Ref. 18, it was found that the FLEET signal intensity was roughly proportional to density over the range of conditions studied. Thus to get a notional sense of what the magnitude of the signal variation should be, the density (based on the measured velocity, total pressure, and total temperature assuming a constant total enthalpy) is plotted in Fig. 6a with the measured intensity. The expected deficit is considerably smaller than that seen in the actual signal; the deficit in the signal intensity is seen to be nearly 40 percent, while that of the density is only 4 percent. To further observe the effects of the local flow conditions, the signal lifetime was calculated within this same profile and is plotted in Fig. 6b. In this same region, the lifetime is decreased from the freestream value by over an order of magnitude.

Understanding this behavior is very difficult without targeted experiments, which these tests were not intended to be. However, there is some information to consider. First, it is unclear whether the actual density effect is or should be visible within the wake region. The precision of the density measurement in Ref. 18 was found to be about 10 percent, which implies that the measurement may not be sensitive enough to resolve the minor density drop within this region of the wake. Second, based on the lifetime measurements it is clear that the signal intensity is being adversely affected by the shortened lifetime. That is, with a reduction in lifetime of over an order of magnitude, it is likely that a large portion of the signal has been lost before the intensifier acquires even the first image. Third, the change in lifetime does not correlate well with any of the mean strain rates or velocity gradients. There is a weak correlation between the magnitude of the transverse velocity fluctuations and the decrease in lifetime, but the trend does not hold from profile to profile. Instead, the current hypothesis is that the loss in signal lifetime is a function not of the observable gradients, but the instantaneous out-of-plane velocity gradients, which could not be resolved but work to fragment the FLEET excitation volume. Measurements made inside a sweeping jet actuator ${ }^{20}$, which locally exhibit similar velocities and flow gradients to those seen within this wake, were not seen to suffer from the loss in signal lifetime, though the FLEET signal was broken up by the strong local gradients. This flow was nominally twodimensional, which suggests that the out-of-plane behavior is likely responsible for the observed trend in lifetime. In the airfoil wake flows, which have a much more three-dimensional character, the decrease in lifetime is almost always accompanied by the loss of cohesion within the FLEET signal. More work needs to be done to ascertain the mechanism responsible for this effect, but for this study making note of the consequences is sufficient.

\section{Velocity Dynamic Range}

In principle, the dynamic range of the velocity measurements should be precision-limited at low velocities and limited by the field of view at higher velocities. That is, when the measured displacements are small due to low velocities and/or a small temporal displacement, the ability to resolve such displacements is nominally dependent on the precision of the measurement algorithm (be it surface fitting or cross-correlation). Likewise at higher velocities, the limit to what can be resolved is imposed by the allowable size of the region of interest on the sensor, since the measurements are imaging based. The initial studies done in the freestream of the $0.3-\mathrm{m}$ TCT facility seem verify these behaviors. ${ }^{17}$ However, with FLEET there are other practical issues that affect the measurement dynamic range.

Consider the flow in the wake of the airfoil; at high Mach numbers and angles of attack, the recirculation region that is present near the trailing edge of the airfoil has influence on the velocity field some distance into the wake. Consequently, the velocities experienced in these regions range from high transonic (near Mach one) to nearly -100 $\mathrm{m} / \mathrm{s}$. Sample probability density functions (PDFs) from several different regions are shown in Fig. 7(b-e) for reference. Per the discussion above, it would suggest that the large positive and negative velocities would be resolvable to the same degree since the measured displacements would be similar. However, this was not the case in practice for a couple of reasons. First among these is the dependence of the signal lifetime on the local strain rates, which was described in Section IV.A. While this region couldn't be formally considered separated flow, it is still subject to exceedingly high local strain rates and unsteadiness in velocity. Consequently, when a velocity approaching $0 \mathrm{~m} / \mathrm{s}$ or below was encountered, the lifetime of the signal tended to be very short and thus reduced the precision of the measurement, making low and negative velocities more difficult to resolve independent of the magnitude of the displacement. Most of the velocities measured in these regions were evaluated using the point-to-point measurement scheme, since rarely was the signal of sufficient duration to use one of the alternative methods. To demonstrate this trend, the fraction of selected velocities in this wake profile are shown in Fig. 7f. As is shown, regions which have

American Institute of Aeronautics and Astronautics 

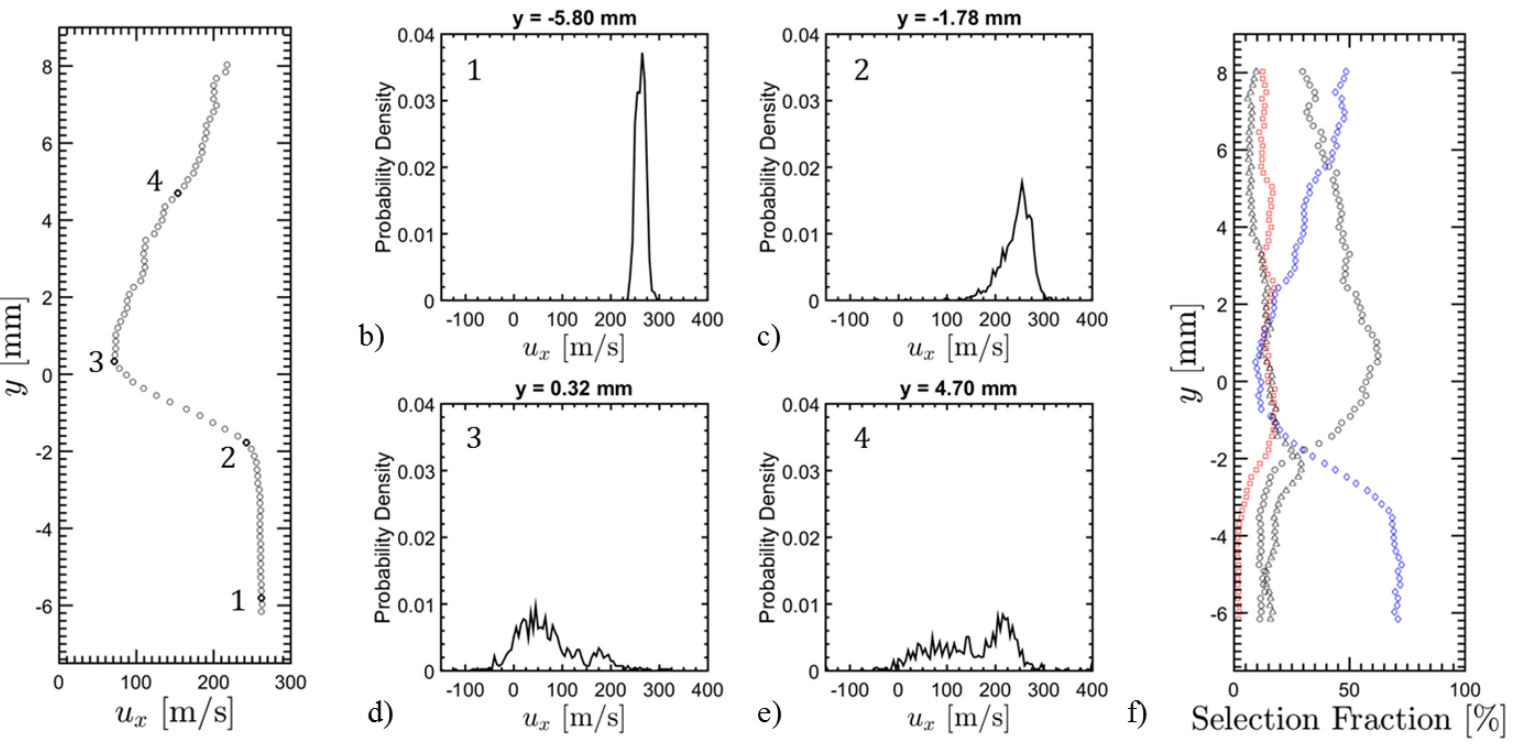

- point-to-point (2 point) 。 linear (3 point) • linear (4 point) $\Delta$ polynomial (4 point)

Figure 7. Several sample velocity probability density functions at different locations in wake behind transonic airfoil. Test conditions were Mach 0.85, $P_{t}=125 \mathrm{kPa}, T_{t}=280 \mathrm{~K}$, and $\mathrm{AoA}=7^{\circ}$. a) Sample velocity profile, b)-e) velocity PDFs corresponding to the labels in $a$ ), and $f$ ) selection fraction for different velocity evaluation distance as function of position in wake.

higher velocities almost universally prefer the fitting methods, and thus were more readily resolvable. An associated effect related to the unsteadiness/lifetime coupling is the loss of signal. That is, in addition to losing precision, the signal could potentially drop below the detection threshold in these same regions, making the measurement of velocities impossible. Thus in practice, the velocity dynamic range is limited toward low and negative velocities because the physics of the FLEET technique cause a dramatic loss of signal in these regions that can reduce measurement precision or altogether inhibit the ability to make a velocity measurement.

At high positive velocities, there does not appear to be any significant limitation in the dynamic range. One concern is the size of the camera field-of-view (FOV). If there are very high velocities, the limited FOV required to operate at high frame rates with the CMOS sensor might limit the observable displacement of the FLEET spot. For example, the camera system used in these studies could maintain a resolution of $240 \mathrm{px} \times 128 \mathrm{px}$ at $100 \mathrm{kHz}$. However, this resolution would still be able to resolve single displacements (given the magnification at the sensor) of nearly 1200 $\mathrm{m} / \mathrm{s}$, which is well beyond what is expected in this facility. Nonetheless, to ensure the highest dynamic range possible, the variable intensifier gating (described in Section II.D) was used. By using varying time delays within a single burst (first a short delay followed by two longer delays), not only are high velocities resolvable, but most instances where the signal lifetime has fallen away due to the strain/unsteadiness issue can still be captured (if only for two frames).

\section{Measurement Accuracy and Precision}

For these experiments, it is assumed that the measurement accuracy is bracketed by the results encountered in previous experiments. That is, measurements are accurate to within 1 to 1.5 percent. Because of the modification to the spatial calibration procedure, it is likely that the measurements lie at the higher-accuracy end of this range, but due to the applied nature of these tests there was no basis for comparison as there had been in other tests. These levels of accuracy were repeatable, and there is no cause to think the accuracy would be worse in these studies. The accuracy will be discussed further in the context of overall measurement uncertainties in Section IV.A.5, but it is being stated here for clarity.

The measurement precision is a much more complicated issue in these experiments than in previous. The reasons for this observation are several. First, as noted in Section IV.A, the lifetime of the FLEET signal is a function of the local instantaneous strain rate of the gas. The shortened lifetime is relevant to the precision because the lifetime of the FLEET signal directly corresponds to the number of visible frames of data are available for evaluation, which is inversely proportional to the precision of the measurement. That is, the more frames of data available, the higher (better) the measurement precision. The implication is that rather than having a fixed measurement precision, there is a spatial variation in the precision within each velocity profile. To quantify this to a greater degree, FLEET data taken

American Institute of Aeronautics and Astronautics 
in static $\mathrm{N}_{2}$ were evaluated at the different time intervals and used to assess the measured velocity fluctuations. Here the precision is defined as one standard deviation of the measured velocity distribution. The results of these studies are shown in Table 1 for each of the three velocity evaluation methods and the combined optimal assessment. As is shown, the precisions are comparable to previous studies using FLEET. ${ }^{19}$ Note that the slightly lower precision in the linear fitting method was the result of having fewer points and a shorter time delay included in the calculation. Additionally, these measurements are taken in static gas, which has shown repeatedly to increase the observed standard deviation in velocity by a factor of nearly 2 either due to contamination by energetically parasitic molecules generated during excitation or by the shorter signal lifetime caused by the former. Also note that the 'optimal' method precision is influenced by the number of samples taken from the individual methods. In the static gas, the point-to-point method is likely to be favored, which is why these precisions are so comparable. Thus in practice the measurement precision is likely to improve over these wind-off values.

Table 1. Wind-off velocity precision measurements for each evaluation method.

\begin{tabular}{lccc} 
& & Time Delay & \\
Method & $\sigma_{u_{x}}(4 \mu s)[\mathrm{m} / \mathrm{s}]$ & $\sigma_{u_{x}}(14 \mu s)[\mathrm{m} / \mathrm{s}]$ & $\sigma_{u_{x}}(24 \mu s)[\mathrm{m} / \mathrm{s}]$ \\
\hline Point-to-point & 4.36 & $\mathrm{n} / \mathrm{a}$ & $\mathrm{n} / \mathrm{a}$ \\
Linear fit & $\mathrm{n} / \mathrm{a}$ & 2.62 & 0.95 \\
Polynomial fit & $\mathrm{n} / \mathrm{a}$ & $\mathrm{n} / \mathrm{a}$ & 4.63 \\
Optimal & $\mathrm{n} / \mathrm{a}$ & $\mathrm{n} / \mathrm{a}$ & 4.18 \\
\hline
\end{tabular}

\section{Spatial Resolution}

In discussing the spatial resolution of the measurements, it is important to distinguish between transverse and stream-wise spatial resolutions, which are limited by factors both common and contrasting. The transverse spatial resolution of the scanning measurements is limited by two primary factors. The first of these is the size of the FLEET spot. Since the cross-correlation algorithm (or the surface fitting algorithm implemented previously), treats the FLEET spot as a single fluid element, all phenomena occurring at length scales within this focal volume get averaged together into a single displacement. In this way, even if the FLEET spot were scanned in very fine increments, discerning independent velocity features at a smaller scale than that of the FLEET spot would be an impossibility for this type of measurement. For this reason, the limiting resolution for the sampling volumes used to construct the velocity profiles was assumed to be the mean $1 / e^{2}$ diameter of the FLEET spot $\left(d_{F L E E T}\right)$, which was approximately $0.7 \mathrm{~mm}$ in the current experiment.

The other factor which influences the transverse spatial resolution of the velocity measurements is the perceived motion of the FLEET spot. For the sampling method described in Section III.E, the primary limitation comes from the vibration of the optical system during the measurement. Specifically, if the initial position of the FLEET spot were moving due to vibration of the laser optical path, camera, or both, the measurements happening at what should nominally be a single point (within the precision of the surface fitting algorithm) would appear to be spread out over some region. Another way to look at this is that there is an uncertainty in the static positioning of the FLEET spot due to vibrations that limits how small of a region can be observed independently. To quantify this phenomenon for each transverse scan, the nominal mean position at each sampling time was subtracted from the instantaneous transverse position, and the limit on the spatial resolution was assumed to be the $2 \sigma_{v}$ offset from the mean position after this process. For all datasets, the peak observed oscillatory magnitude $\left(2 \sigma_{v}\right)$ was $0.4 \mathrm{~mm}$, which is of similar magnitude to the size of the FLEET spot. This figure is also significantly reduced in comparison to previous tests, which had $1 \sigma_{v}$ magnitudes up to nearly $1 \mathrm{~mm} .{ }^{19}$ The likely cause of this improvement is the smaller camera that was used during testing, which reduced the cantilevered load on the mounting platform and helped to minimize the overall deflection of the system.

Assessing the cumulative effect on the transverse spatial resolution is dependent on the interpretation of the observed vibrations. That is, whether the vibrations are primarily affecting the laser beam, camera, or both changes how the two primary limitations interact. In the case of the laser beam being the primary vibrating component, the vibrations essentially have no effect on the spatial resolution. The FLEET signal appears where it is supposed to in both the lab and camera frames-of-reference, and thus the lower limit on the transverse spatial resolution is the averaging over the area of the FLEET signal. In the second case, where it is primarily the camera system vibrating and the laser is scanning without vibration, the vibrational amplitude has a significant effect. Since the camera frameof-reference is now shifting relative to the lab frame-of-reference, numerous spatial transverse positions can fall within a sampling region. To quantify this effect, consider first a measurement volume made at the spot-size limited resolution, $0.7 \mathrm{~mm}$. The transverse scan rate of the laser system was approximately $0.07 \mathrm{~mm} / \mathrm{s}(10 \mathrm{~s}$ to $\mathrm{scan}$ through

American Institute of Aeronautics and Astronautics 
the volume). Since the laser is oscillating with a magnitude of up to $2 \sigma_{v}$, the fixed measurement region in the camera frame-of-reference will see spots originating up to $5.7 \mathrm{~s}$ before and after the primary scan interval. Converting back to spatial units, this means that the $0.7 \mathrm{~mm}$ measurement region will have an effective resolution of $1.5 \mathrm{~mm}$ in the lab frame-of-reference. Unfortunately, with these measurements there is no real way to tell which interpretation is correct, since there are no visual cues in the images to discern what the lab frame-of-reference is relative to the camera. In all likelihood, the true lower limit is somewhere between these two extremes. Nonetheless, these two figures of $0.7 \mathrm{~mm}$ and $1.5 \mathrm{~mm}$ serve as the potential lower-bounds to the spatial resolution. The sample volume size was selected to be $0.7 \mathrm{~mm}$ during the data analysis, but it must be understood that the true resolution might be over twice that dimension when both of these effects are considered.

The stream-wise spatial resolution is limited by both the size of the FLEET spot, the local velocity, and the temporal delay between successive frames. Much like the transverse spatial resolution, the FLEET spot size poses a limit on the spatial resolution by creating an averaging effect in the region surrounding the measured centroid location. The local velocity and temporal separation affect the amount the FLEET spot advects. Consequently, length scales under twice the advective distance cannot be resolved. A more thorough discussion of this effect can be found in Ref. 19.

\section{Overall Measurement Uncertainty}

The overall uncertainty in the instantaneous velocity measurements is dictated by numerous factors. Among these are what are termed procedural uncertainties, which relate to how the experiment was set up and conducted, and analytical uncertainties, which result from the data analysis. A comprehensive breakdown and calculation of each of the constituent uncertainties is given in Appendix A, but a summary of each uncertainty considered and a synopsis of the results is given here. The procedural uncertainties include a number of separate effects. The uncertainty caused by the large depth-of-field of the camera/lens system and the associated variation in magnification is the most substantial contributor to the overall uncertainty. Due to this effect, a 4 percent uncertainty is present in every velocity measurement, regardless of its magnitude simply because the optimum location of the calibration target cannot be determined precisely. Moreover, because the position of the laser beam and the tunnel itself change from day to day and recalibration is often not possible, the optimal target pattern position can change without any indication in the collected images. The second primary procedural uncertainty is the placement of the calibration target and the camera system relative to the measurement location. To specify, there is a potential that the calibration target was placed into the test section at a small angle relative to the primary axes of the tunnel. Likewise the camera could potentially be rotated relative to the flow, and finally the camera and calibration target might have rotated with respect to the flow and each other. These effects also then incur uncertainties resulting from out-of-plane velocities which could not be resolved. For a full analysis of these different uncertainties, see Appendix A.

It will suffice to say here that the only relevant situation to consider in this particular set of experiments is that corresponding to the camera and dotcard being jointly rotated with respect to the flow or tunnel axes. This uncertainty is dependent on the magnitude of the measured velocity and the relative direction of the flow. When rotation about the $y$-axis is considered (see Fig. 8a), the uncertainty that arises in the stream-wise velocity is of the order 0.1 percent, independent of velocity magnitude (the actual magnitude of the velocity uncertainty varies from 0 to $0.65 \mathrm{~m} / \mathrm{s}$ for velocities ranging from 0 to $500 \mathrm{~m} / \mathrm{s}$, respectively. However, when the presence of out-of-plane velocities are then considered, this uncertainty becomes notably larger depending on the magnitude of the out-of-plane velocity. For most of the measurement locations considered in these studies, the uncertainty is below 1 percent with larger uncertainties occurring at lower velocities. Similar trends are observed if rotation about the $x$-axis is considered, with the transverse velocity having a baseline uncertainty of roughly 0.1 percent as well. However, the additional uncertainty of out-ofplane velocities has a much greater effect on the transverse component of velocity due to the lower overall magnitude (see Fig. 8b). Most transverse velocities measured in these tests fall between a 1 and 10 percent uncertainty. Finally, considering rotation about the $z$-axis, the transverse and stream-wise velocity magnitudes jointly affect one another. A map of these two uncertainties can be seen in Fig. 9a and 9b. The stream-wise velocities have an uncertainty at or below 1 percent for most of the measurement range, while the transverse velocity is affected more severely, again due to its smaller magnitude, with uncertainties lying between 10 and 20 percent over most of the observed measurement domain. These effects are coupled, and must be compounded when determining the overall uncertainty.

American Institute of Aeronautics and Astronautics 

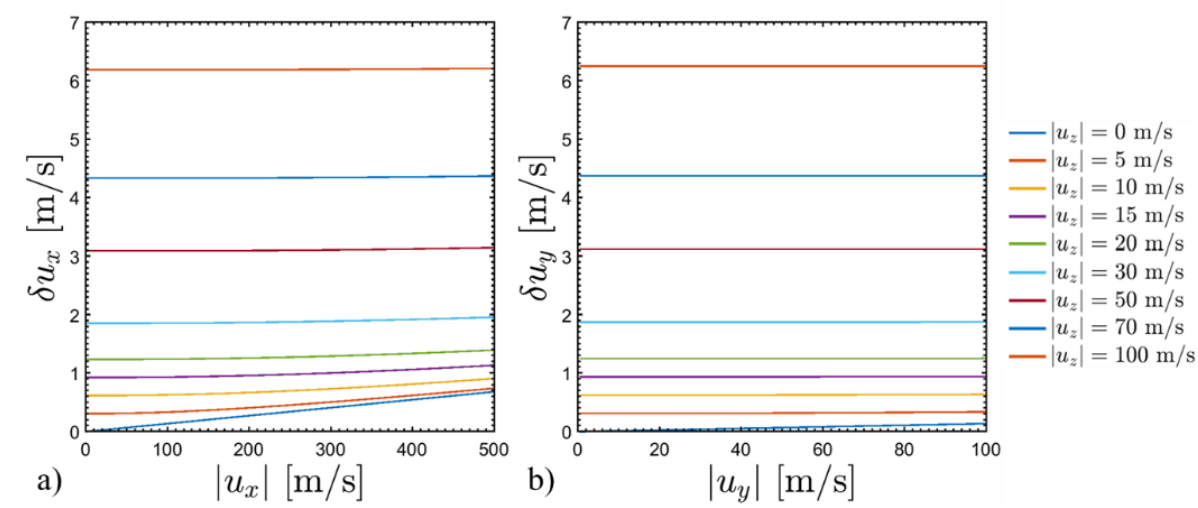

Figure 8. Velocity uncertainty caused by out-of-plane camera rotation and out-of-plane velocity. a) streamwise velocity and $b$ ) transverse velocity.

Analytical uncertainties arise from the data processing procedures used to extract velocity information from the raw image data. While the list of such uncertainties is extensive, the two primary uncertainties arise from either a) the cross-correlation or surface fitting algorithm used in evaluating displacements or b) the method used to calculate velocities from the position data. Regarding the former point, the uncertainty of the adaptive cross-correlation algorithm used in these studies was found to be on the order $0.1 \mathrm{px}$, or approximately $12 \mu \mathrm{m}$ at the current magnification. The precision was actually higher as the SRR increased, but the $0.1 \mathrm{px}$ was the minimum observed precision for the solver. The uncertainty that arises from the velocity evaluation method varies from method to method. For the point-to-point method, this uncertainty is a function of displacement uncertainty as established by the precision of the cross-correlation algorithm as well as that of the temporal delay. Regarding the linear and polynomial fitting methods, these methods have an uncertainty associated with each of the fit parameters considered, one of which is the velocity. For the samples that were adequately described in this, this error was often small in magnitude, around 0.5 $\mathrm{m} / \mathrm{s}$ at the lowest velocities (and smaller at higher velocities).

Putting all of this information together, there is no simple uncertainty that can be directly applied to the instantaneous FLEET velocities in all circumstances. For the stream-wise velocities, the variable magnification was the primary source of uncertainty. Cumulative uncertainties ranging from 4.1 to 5.3 percent (at $1 \sigma$ levels) are apparent at high to low velocities, respectively. The transverse velocity, while subject to the same calibration uncertainty due to the variable magnification is also subject to greater uncertainty due to camera rotations than the stream-wise component due to its smaller magnitude. Cumulative uncertainties in the transverse velocities instead peak between 12 and $15 \mathrm{~m} / \mathrm{s}$, which can equal or exceed the measured velocity in some circumstances. Finally, comparing the measurement accuracy found in previous studies (approximately 1 percent) to the uncertainties, the uncertainty values are found to be considerably larger. By definition, the uncertainty is meant to bound the measured value. The

a)

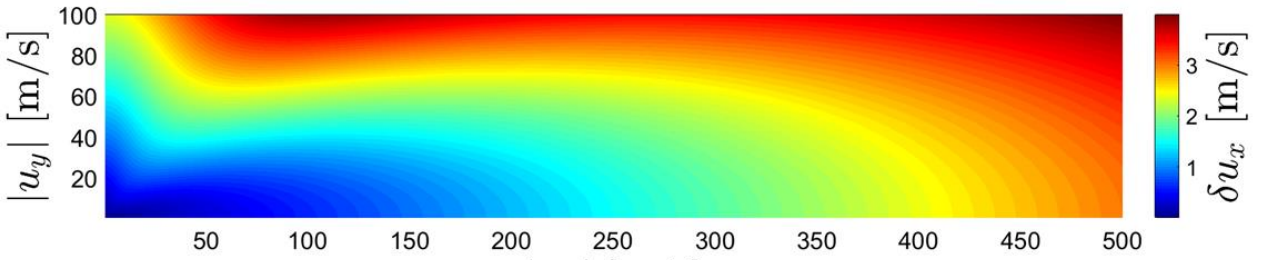

$\left|u_{x}\right|[\mathrm{m} / \mathrm{s}]$

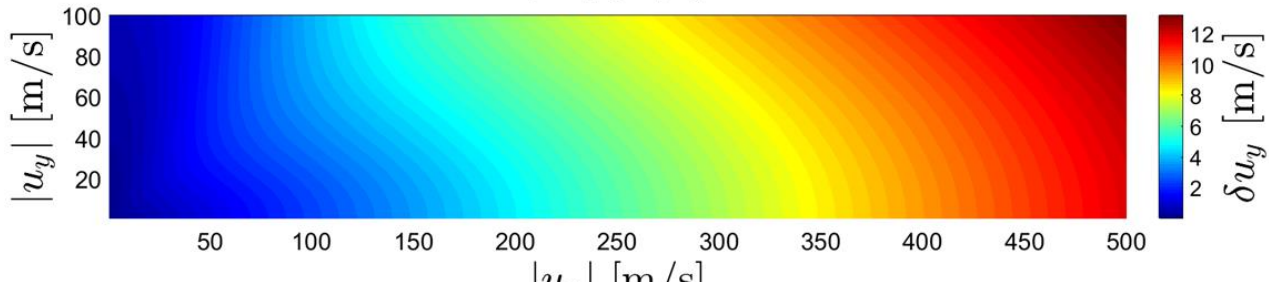

b)

$$
\left|u_{x}\right|[\mathrm{m} / \mathrm{s}]
$$

Figure 9. Velocity uncertainty caused by in-plane rotation of the camera. a) stream-wise velocity and b) transverse velocity.

American Institute of Aeronautics and Astronautics 
measurement may very well be far more accurate than the uncertainty suggests, but there is often no way of knowing without a basis for comparison. While previous tests were afforded this advantage, the present tests demonstrated for the first time the level of uncertainty that potentially must be considered in a real testing environment.

\section{B. Flowfield Analysis}

Several different features of the airfoil flowfields are discussed in this section including general observations about the flowfield, the wake region downstream of the airfoil, and evidence observed of flow separation. The measurements focus primarily on the highest Mach number cases since these are more representative of the transonic regime.

\section{Wall pressure distributions}

Tunnel wall pressure distributions for two Mach 0.85 flowfields are show in Fig. 10, shown with respect to the airfoil, indicating the pressures at three different span-wise locations. The first of these shown in Fig. 10a for a 0degree $A o A$ indicates a relatively minimal perturbation to the flowfield by the airfoil. There is a slight rise in pressure due to the influence of the stagnation region slightly upstream of the airfoil $(x \in[-100,0])$, followed by the expected drop in pressure over the surface of the airfoil accompanying the acceleration of the flow. The peak Mach number based on the wall pressure measurements in this region is just over 0.87, further indicative of the small perturbation to the flow. Another crucial point to make regarding the 0-degree $A o A$ flowfield is the spatial uniformity over the surface of the airfoil. The pressures for all three span-wise locations vary by only 0.3 percent on the top wall and 1.2 percent on the bottom wall. While not indicative of the behavior of the flowfield as a whole, it does suggest that there is minimal crossflow behavior in this flowfield configuration. Finally, the pressures in the duct recover and exceed the freestream pressure following the interaction with the airfoil. Previous experiments done with FLEET in this facility utilized physically parallel walls, which resulted in a fairly constant favorable pressure gradient along length of the duct. The aerodynamically parallel walls, which were used to create more clearance for the model and allowed operation at higher Mach numbers, result in the formation of this adverse pressure gradient and gradual deceleration of the flow under certain operating conditions. The pressure data also suggests that there is a slight asymmetry in the mounting of the airfoil, evidenced by the slight variations seen between the top and bottom wall pressure distributions. Specifically, it is likely that the airfoil was at a slight $A o A$ (less than 1 degree), which will be further demonstrated in the measured velocity profiles in Section IV.B.2.

Consider next the flowfield at the same Mach number but a higher $A o A$ (7 degrees). The drastic differences in the flowfield are apparent from the pressure distributions. Notably, there is a larger pressure rise observed on the compressive side of the airfoil along with a slightly wider discrepancy between the pressures along the span-wise direction. On the expansion surface, a steep drop in pressures is observed over the surface of the airfoil, with a much larger pressure drop occurring near the wall on which the airfoil is mounted due to the greater thickness. This drop in pressure is then followed by a fairly steep rise in pressure toward the trailing edge of the airfoil due to either simple pressure recovery or perhaps a weak compression wave. Note also the significant non-uniformity across the span of the tunnel, with pressures varying on the order of 10 percent. This behavior is evidence for a strong threedimension character to the flowfield.
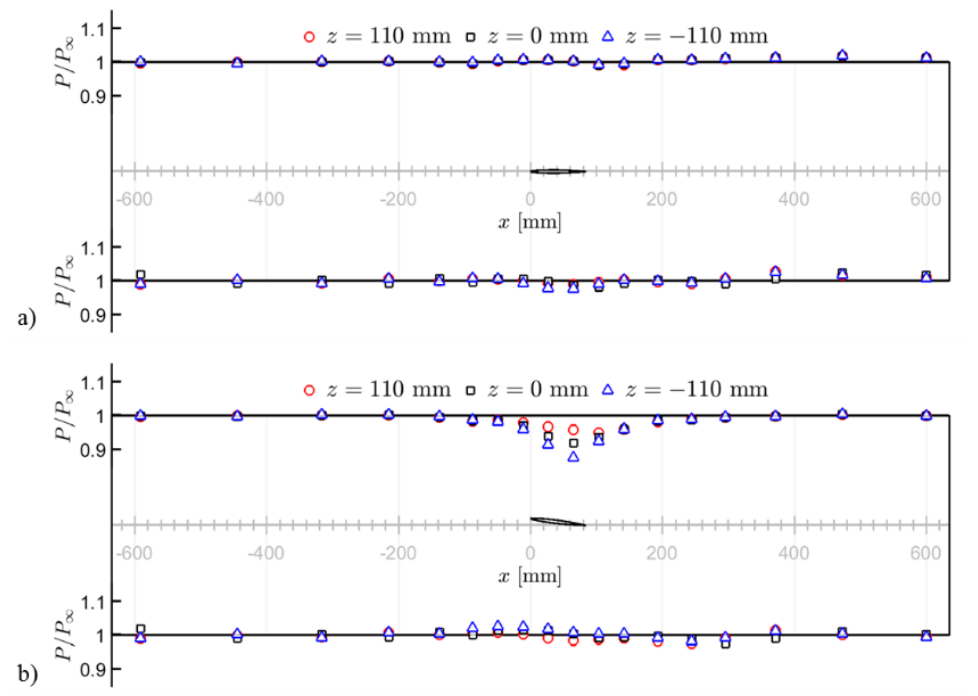

Figure 10. Tunnel top and bottom wall pressure distributions relative to airfoil position. a) Mach $0.85, P_{t}=125 \mathrm{kPa}, T_{t}=280 \mathrm{~K}$, and $\mathrm{AoA}=0^{\circ}$ and b) Mach 0.85, $P_{t}=125 \mathrm{kPa}, T_{t}=280 \mathrm{~K}$, and $\mathrm{AoA}=7^{\circ}$.

\section{Wake measurements}

Velocity profiles taken in the Mach 0.85, 0-degree AoA flowfield are shown in Fig.11, showing the stream-wise and transverse velocities and their fluctuations. These profiles were taken $4.5 \mathrm{~mm}$ downstream of the trailing edge of the airfoil. The FLEET velocity measurements indicate uniformity in the velocity on both surfaces of the airfoil, approaching $260 \mathrm{~m} / \mathrm{s}$. The observed velocity deficit in the stream-wise velocity component (Fig. 11a) is fairly narrow

American Institute of Aeronautics and Astronautics 


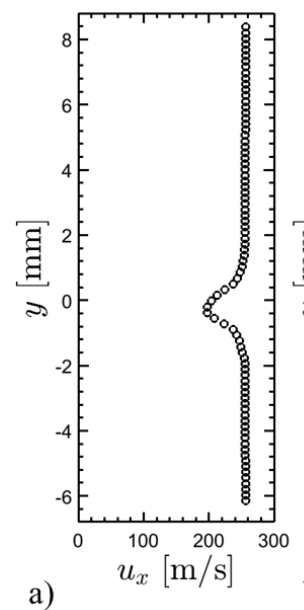

a)

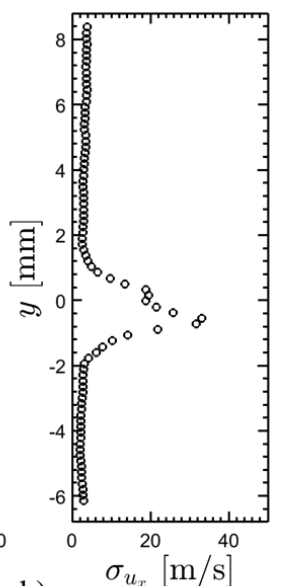

b)

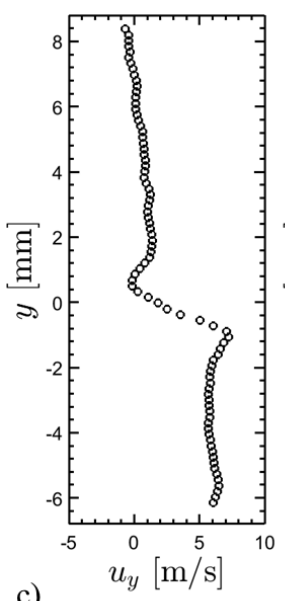

c)

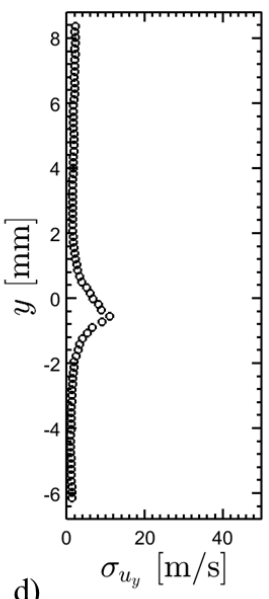

d)

Figure 11. Velocity profiles at $\boldsymbol{x}=\mathbf{8 8 . 4} \mathbf{~ m m}$ (wake) and $\boldsymbol{A o A}=\mathbf{0}^{\circ}$. a) mean stream-wise velocity, b) fluctuating stream-wise velocity, c) mean transverse velocity, and d) fluctuating transverse velocity. Conditions are Mach 0.85, $P_{t}=125 \mathrm{kPa}$, and $\mathrm{T}_{t}=$ $280 \mathrm{~K}$.

at this location and has velocities as low as $180 \mathrm{~m} / \mathrm{s}$ in the mean. The wake is seen to be approximately $2 \mathrm{~mm}$ in transverse extent at this location. The stream-wise velocity fluctuations are seen to peak within either shear layer surrounding the wake. This result indicates that the local measurement precision is sufficiently low in magnitude to resolve legitimate velocity fluctuations in this region. The peak precision sitting around $4 \mathrm{~m} / \mathrm{s}$, the velocity fluctuations are of sufficiently high magnitude to be visible. The bottom shear layer is seen to have notably higher fluctuations than the top surface, which is indicative of a slight $A o A$ at this condition. The effect is mirrored in the transverse velocities (Fig. 11c), which show anti-symmetry across the transverse centerline, but with a centerline shift of $2 \mathrm{~m} / \mathrm{s}$, which corresponds to a flow angle of 0.6 degrees in this region. The transverse velocity fluctuations (Fig. 11d) reveal a peak in this same location, further indicating the likelihood of the slight AoA in the positioning of the airfoil. As mentioned in Section IV.B.1, a slight asymmetry was noticed in the pressure distributions on the top and bottom walls as well, which is consistent with the airfoil sitting at a slight angle of attack. Considering the uncertainty in velocity described above in Section IV.1.E above, there is a nearly $6 \mathrm{~m} / \mathrm{s}$ uncertainty in the velocity in this region, so it is also possible that this observation is simply an artefact of the measurement process. Though omitted here for brevity, the wake profiles at lower operating Mach numbers did not show such asymmetry indicating that the higher dynamic pressure in the tunnel might have caused a slight shift in the angle of the airfoil or turntable due to the increased aerodynamic load.

Velocity profiles at a further downstream location within this same flowfield are shown in Fig. 12, taken $19 \mathrm{~mm}$ downstream of the airfoil. The growth of the wake are apparent in the stream-wise velocity profile (Fig. 12a). Here, the magnitude of the velocity deficit was seen to decrease from 80 to $55 \mathrm{~m} / \mathrm{s}$, while the transverse extent has grown to $2.4 \mathrm{~mm}$. The stream-wise velocity fluctuations (Fig. 12b) are observed to have decreased in magnitude and spread out transversely as well. The obvious asymmetry that was observed upstream is no longer apparent in the stream-wise fluctuations; the peak seen in each shear layer is of similar magnitude. This change in behavior is likely the result of a repositioning of the airfoil. While making measurements, the $A o A$ and Mach number were all varied before moving the measurement location downstream. Consequently, the airfoil has been repeatedly moved through numerous angles of attack before being repositioned between these two data runs shown in Fig. 11 and 12. It is therefore likely that the $A o A$ is slightly different between these runs, resulting in an asymmetry in one profile and not the other. Nonetheless, the FLEET velocity measurements show ample precision and accuracy to be able to resolve the general fluid mechanical behavior of the flowfield under these operating conditions, sufficient to even notice inconsistencies between runs.

American Institute of Aeronautics and Astronautics 


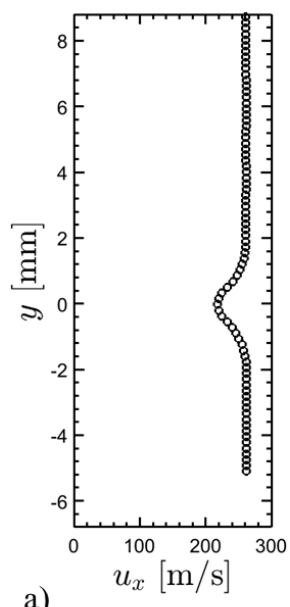

a)

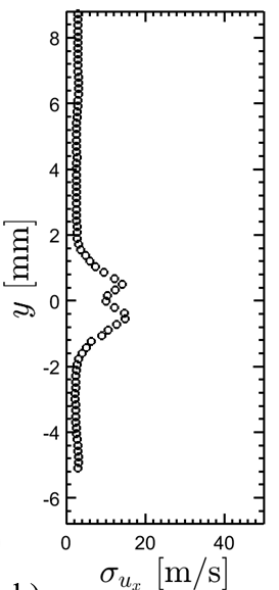

b)

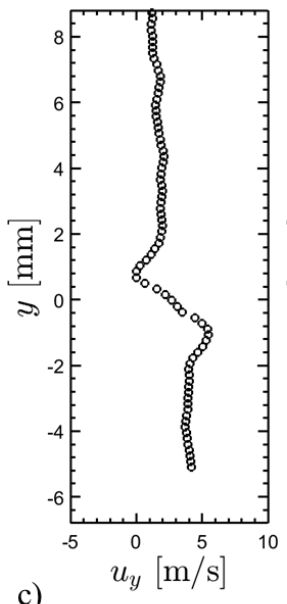

c)

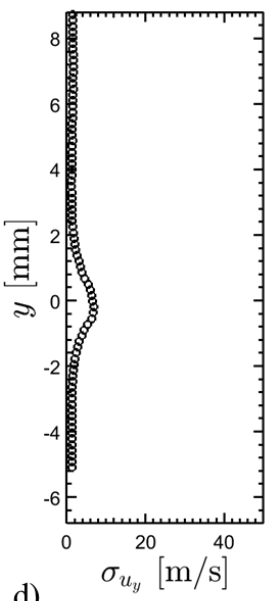

d)

Figure 12. Velocity profiles at $\boldsymbol{x}=\mathbf{1 0 2 . 9} \mathbf{m m}$ (wake) and $\boldsymbol{A o A}=\mathbf{0}^{\circ}$. a) mean stream-wise velocity, $b$ ) fluctuating stream-wise velocity, c) mean transverse velocity, and d) fluctuating transverse velocity. Conditions are Mach 0.85, $P_{t}=125 \mathrm{kPa}$, and $T_{t}=$ $280 \mathrm{~K}$.

\section{Appearance of flow separation}

The velocity profiles indicate the presence of flow separation as the angle of attack of the airfoil was increased. Fig. 13 through 16 show velocity profiles in the same measurement locations of Figs. 11 and 12 at $A o A s$ of $3.5^{\circ}$ and $7^{\circ}$. In the case of the smaller angle of attack (Figs. 13 and 14), there is not a substantial effect on the overall wake structure. A slightly larger velocity deficit is observed in the stream-wise velocity (Fig. 13a and 14a), and the transverse velocities (Figs. 13c and 14c) have shifted to be entirely negative commensurate with the angle of the flow around the airfoil near the trailing edge. The fluctuating stream-wise velocity in the upstream measurement location (Fig. 13b) indicate a less well-defined set of shear layers as with the 0 degree angle of attack case; rather than attaining a maximum in the shear layers, the peak fluctuations appear in the center of the velocity profile. At the downstream measurement location (Fig. 14), the overall wake structure has spread as anticipated, while the large fluctuations in the stream-wise velocity component (Fig. 14b) have lessened in magnitude and organized into two distinct shear layers, though the top shear layer is possessed of higher overall fluctuation content than that on the bottom surface.

At a higher-still angle of attack, 7 degrees, the flowfield has transitioned in character completely, consistent with the stark contrast in wall pressure distribution discussed in Section IV.B.1. The velocity profiles at both the upstream and downstream measurement locations (Figs. 15 and 16, respectively) indicate that the subtle wake structure observed at lower angles of attack has developed several new features. While the flow on the underside of the airfoil at the

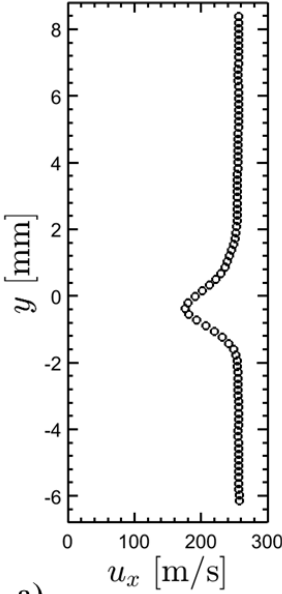

a)

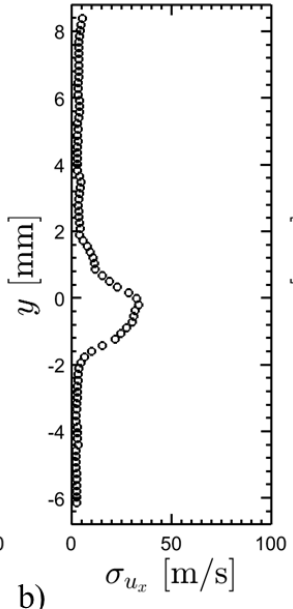

b)

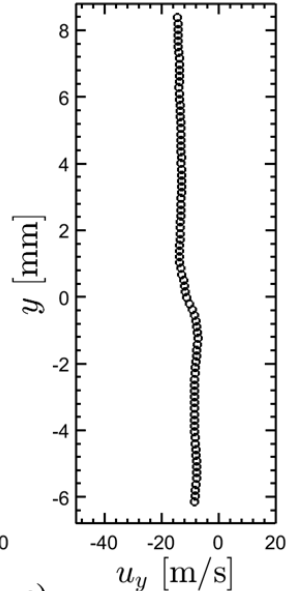

c)

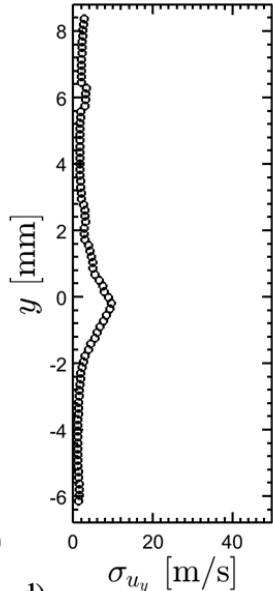

d)

Figure 13. Velocity profiles at $\boldsymbol{x}=\mathbf{8 8 . 4} \mathrm{mm}$ (wake) and $\boldsymbol{A o A}=\mathbf{3 . 5 ^ { \circ }}$. a) mean stream-wise velocity, b) fluctuating streamwise velocity, c) mean transverse velocity, and d) fluctuating transverse velocity. Conditions are Mach $0.85, P_{t}=125 \mathrm{kPa}$, and $T_{t}=280 \mathrm{~K}$

American Institute of Aeronautics and Astronautics 

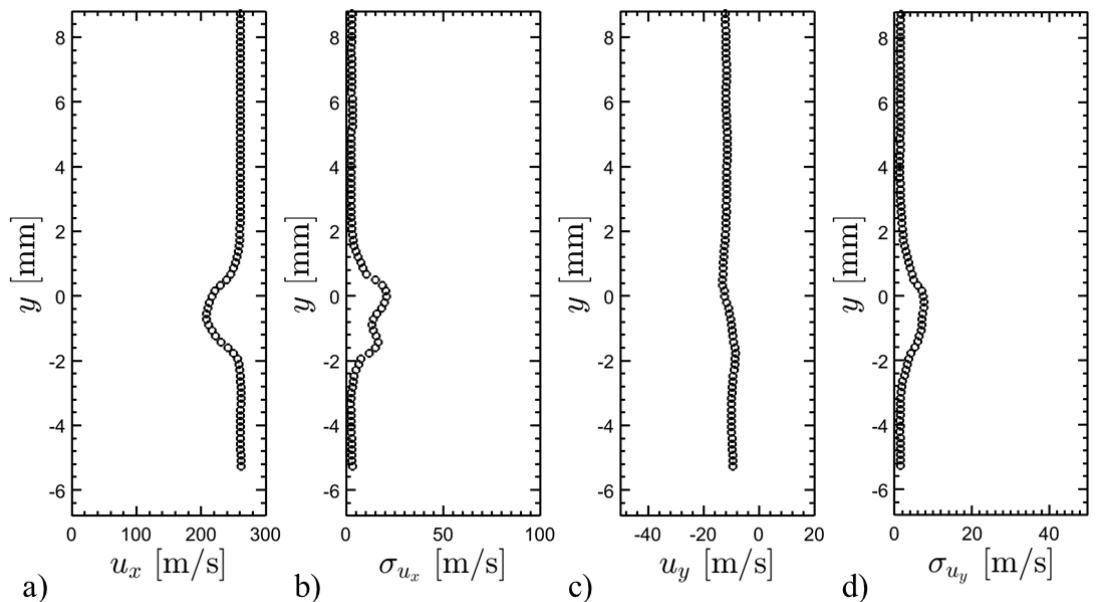

Figure 14. Velocity profiles at $\boldsymbol{x}=\mathbf{1 0 2 . 9} \mathrm{mm}$ (wake) and $\boldsymbol{A o} \boldsymbol{A}=3 . \mathbf{5}^{\circ}$. a) mean stream-wise velocity, $b$ ) fluctuating streamwise velocity, c) mean transverse velocity, and d) fluctuating transverse velocity. Conditions are Mach $0.85, P_{t}=125 \mathrm{kPa}$, and $T_{t}=280 \mathrm{~K}$.
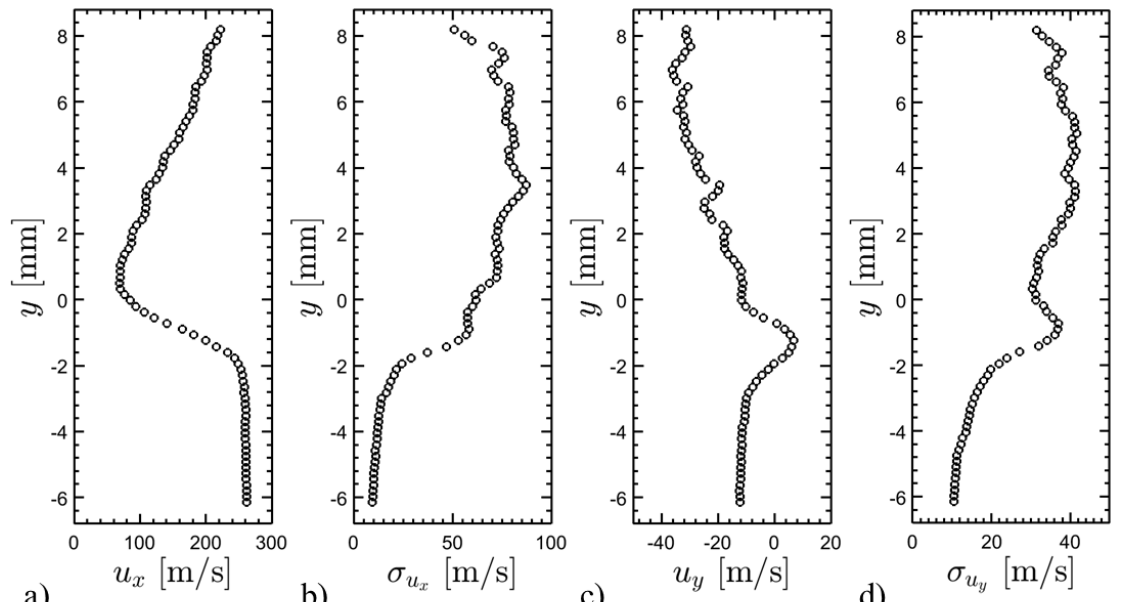

Figure 15. Velocity profiles at $\boldsymbol{x}=\mathbf{8 8 . 4} \mathbf{~ m m}$ (wake) and $\boldsymbol{A o A}=7^{\circ}$. a) mean stream-wise velocity, $b$ ) fluctuating stream-wise velocity, c) mean transverse velocity, and d) fluctuating transverse velocity. Conditions are Mach 0.85, $P_{t}=125 \mathrm{kPa}$, and $T_{t}$ $=280 \mathrm{~K}$.

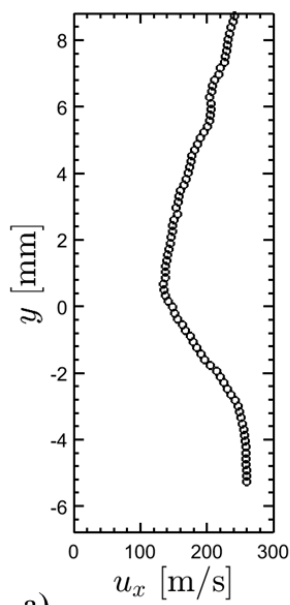

a)

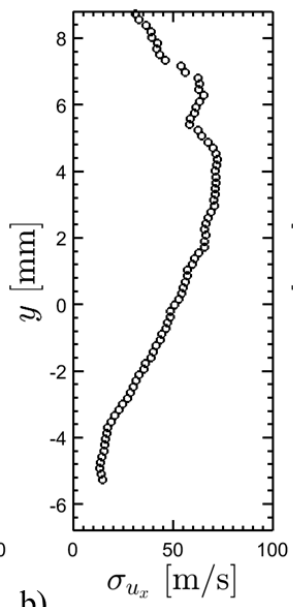

b)

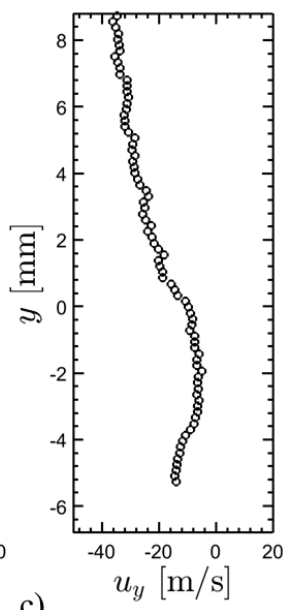

c)

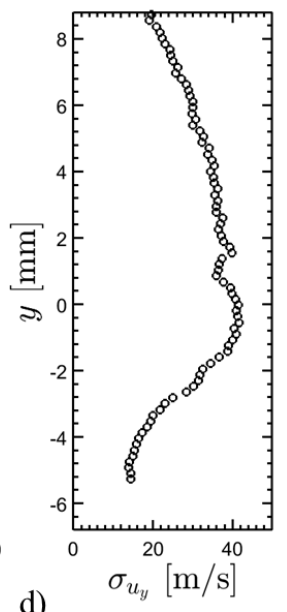

d)

Figure 16. Velocity profiles at $\boldsymbol{x}=\mathbf{1 0 2 . 9} \mathrm{mm}$ (wake) and $\boldsymbol{A o A}=7^{\circ}$. a) mean stream-wise velocity, b) fluctuating streamwise velocity, $c$ ) mean transverse velocity, and d) fluctuating transverse velocity. Conditions are Mach $0.85, P_{t}=125 \mathrm{kPa}$, and $T_{t}=280 \mathrm{~K}$.

American Institute of Aeronautics and Astronautics 
upstream position remains relatively quiescent, the stream-wise profile (Fig. 15a) indicates a much larger velocity deficit, both in physical extent and magnitude. The fluctuating velocities (Figs. 15b and 15d) show a substantial increase in these same regions. PDFs of this wake profile at several locations are shown in Fig. 7a-e. The region now is subject to a large fraction of negative velocities throughout the wake. Note also that within the wake region, some of the velocity PDFs are no longer Gaussian in character, implying that the flow is alternating between different types of behavior consistent with chaotic or intermittently reversed flow. Observation of the downstream measurement location (Fig. 16) indicate that these features persist, while the relatively quiescent region below the airfoil has started to decrease in velocity and increase in fluctuation magnitude due to the growth of the shear layer. Likewise the wake and velocity fluctuations disseminate into the surrounding flow as well by the downstream measurement location.

These observations indicate that unsteady flow separation has occurred upstream near the trailing edge of the airfoil. For this reason, additional measurement locations were evaluated near the trailing edge of the airfoil to try to resolve the features responsible for this change in character of the wake region. Velocity profiles and pseudostreamlines are shown in Fig. 17. A total of 12 different measurement locations were evaluated on this airfoil. The velocity vectors on the compression surface of the airfoil (Fig. 17a) suggest attached flow throughout the measurement region. The small fluctuating velocities (Fig. 17b) observed in the same location further support this notion. The uniform velocity seen on lower region of the wake is consistent with these observations. The top surface is notably different in character. The upstream side of the measurement region sees velocities nearing $500 \mathrm{~m} / \mathrm{s}$, or nearly Mach 2. Since the airfoil is rotating about its trailing edge as the angle of attack is changed, there is a very strong acceleration on the top surface of the airfoil, leading to the supersonic velocities observed. These velocities persist downstream and gradually decrease to the lower velocities seen in the wake measurements. Of particular interest in these measurements are those made on the top surface of the airfoil near the trailing edge. First, the velocities do not seem to follow the curvature of the airfoil as they did on the bottom surface. Second, a strong transverse gradient exists in the velocity as the trailing edge is approached. Many velocities in these regions are near zero and negative. While measurements were not made closer to the surface at this measurement location, these observations along with the drastic change in character undergone by the wake are strong evidence of flow separation. An interesting feature seen in the pseudo-streamlines is the slight 'bump' that appears in the streamlines around $x=67 \mathrm{~mm}$. This feature could be the result of a weak compression wave associated with the onset of flow separation. Although no drastic changes were seen in the stream-wise velocity, the transverse-velocity did suddenly increase in this same region, which might correspond to a weak shock. The large fluctuating velocities present in these regions also match the observations made in the wake, where a broad distribution of velocities were observed throughout the entire area sitting over the airfoil.

\section{Conclusions}

FLEET velocimetry was used to make measurements around an airfoil in a transonic, cryogenic, wind tunnel for the first time. The velocity measurements were found to have a wide dynamic range, though measurements at low and negative velocities were found to be impeded to some extent by a strain- or transient-dependent decrease in the FLEET signal lifetime. The measurement accuracy was similar to previous studies, and the precision of the measurements was found to be dependent on the method used to evaluate the velocity. The spatial resolution of the velocity measurements was a function primarily of the size of the FLEET spot, but was further limited by the vibrations of the tunnel and/or measurement equipment. Finally, the overall measurement uncertainties were assessed and found to lie between 4 and 5 percent in the stream-wise velocity components and up to $15 \mathrm{~m} / \mathrm{s}$ in the transverse velocity component. To demonstrate the velocimetry, measurements were shown in several Mach 0.85 flowfields. The FLEET velocimetry proved of sufficient accuracy, precision, and resolution to measure the velocity deficit and fluctuating velocities within the flowfield. Moreover, features such as shear layer and wake growth, dissemination of velocity fluctuations, and even subtle asymmetries in the flow that developed due to errors in positioning the airfoil were apparent, indicating the overall sensitivity of the FLEET velocity measurements. To further examine the utility of the technique, a flow separation phenomenon was investigated on the same airfoil at a high angle of attack by utilizing numerous measurement locations on the top and bottom surfaces of the airfoil. Through the mean and fluctuating velocity measurements and the construction of pseudo-streamlines, strong evidence was presented that (at least) incipient flow separation was present near the trailing edge of the airfoil, as well as the possibility of a weak compression wave near the onset of the flow separation. These studies thus serve to legitimize the FLEET velocimetry technique for making measurements in applied flows.

American Institute of Aeronautics and Astronautics 
a)

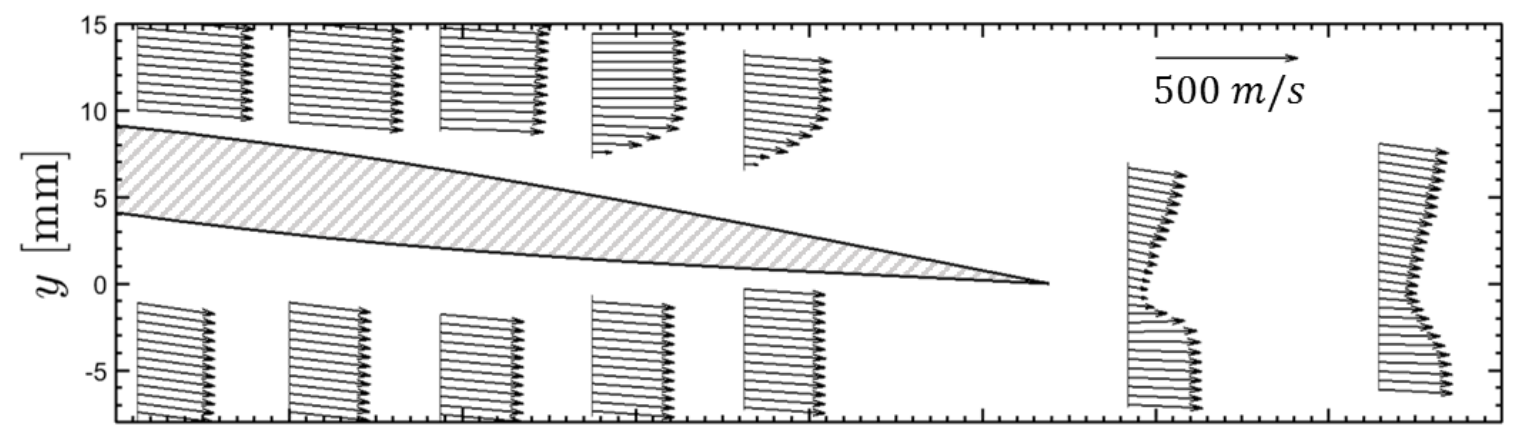

b)

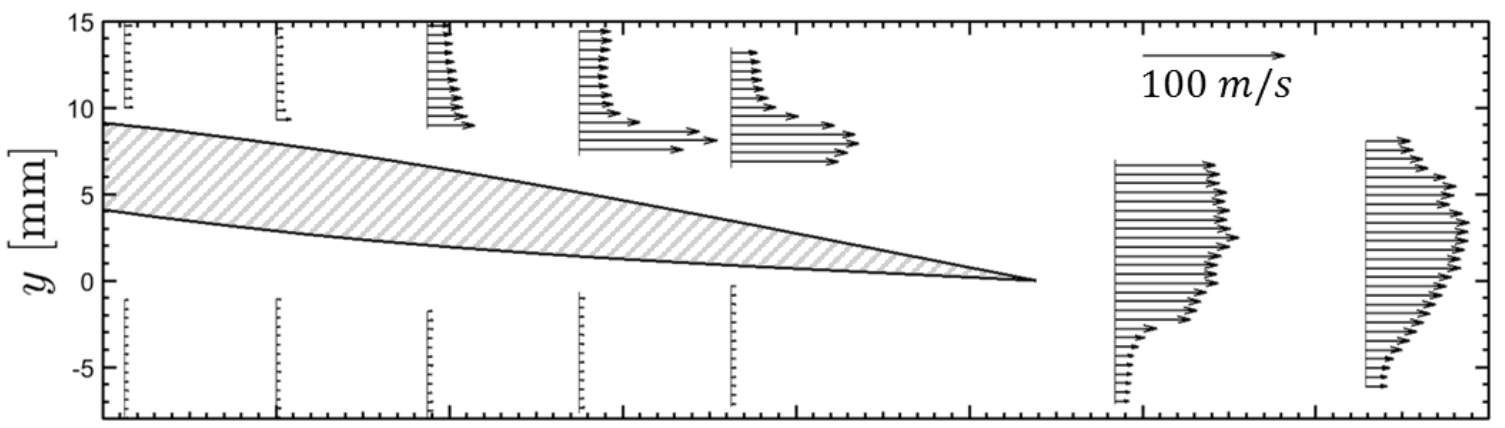

c)

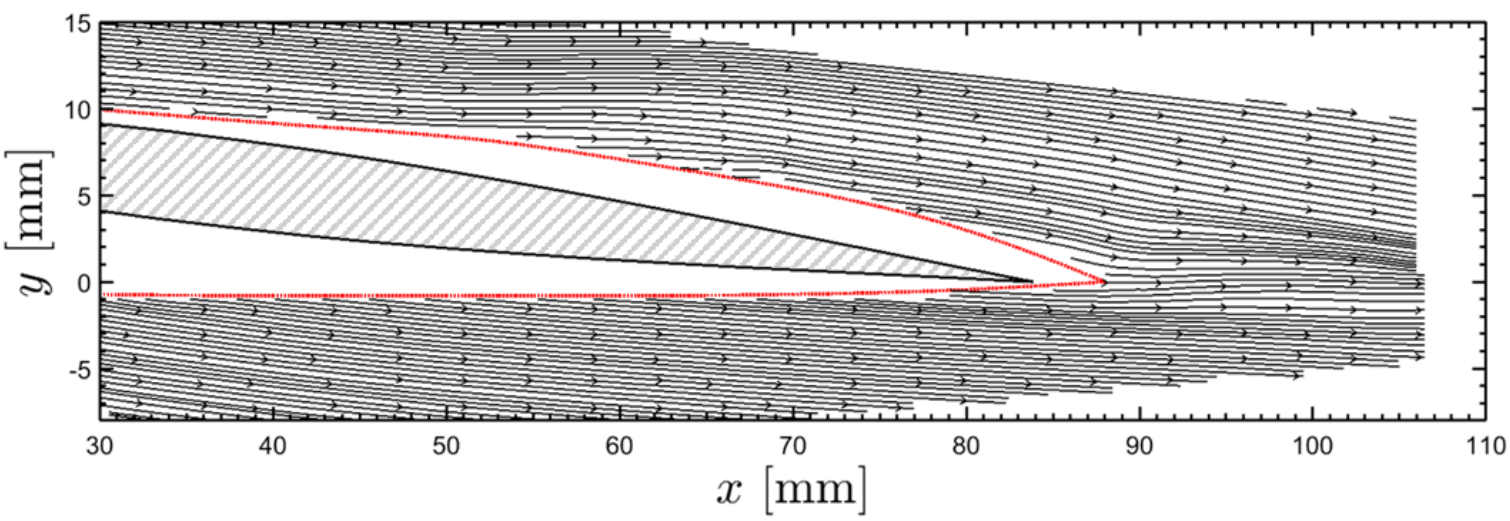

Figure 17. Velocity vectors and pseudo-streamlines over trailing edge and wake of transonic airfoil at Mach 0.85 and $\boldsymbol{A o A}=7^{\circ}$. a) mean velocity vectors, b) fluctuating velocity $\left(\left(\left\langle u^{\prime}\right\rangle^{2}+\left\langle v^{\prime}\right\rangle^{2}\right)^{1 / 2}\right)$, and c) pseudo-streamlines based on mean velocity. Red dashed line indicates where data was present to construct streamlines.

\section{Appendix A. Measurement Uncertainty}

There are numerous uncertainties that need to be considered in determining the overall measurement uncertainty in instantaneous velocity measurements. To be consistent with the discussion in Section IV.A.5, these uncertainties will be divided into procedural uncertainties and analytical uncertainties.

\section{A. Procedural Uncertainties}

\section{Variation in magnification}

As explained in Section II.C, the placement of the calibration target pattern is a large source of uncertainty in these measurements. To explain the procedure used during testing; the FLEET spot was first generated in the tunnel. Then, the camera system was focused on the FLEET signal. Finally, since the target pattern could not be placed into the camera FOV while the laser was still active, the femtosecond laser was inhibited, and the calibration target was placed within the test section until the target pattern appeared as focused as possible on the camera's readout. However, due to the large depth-of-field of the imaging system, the target appears to be in focus for a substantial distance. Thus, it is possible that the target was situated anywhere within the depth-of-field of the imaging system when recorded, leading to a rather significant uncertainty due to calibration. In an attempt to assess this and achieve a higher degree

American Institute of Aeronautics and Astronautics 
of accuracy in the measurements, the target pattern was scanned through the depth-offield during these experiments to both find what might be the optimum position for the focus and assess the uncertainty associated with this variation. The results of one such calibration scan are shown in Fig. A.1. Figure A.1a shows the observed variation in the spot radius as a function of distance away from the camera, while Fig. A.1b shows the magnification at the same locations. A 4percent variation is noted throughout the measured distance within the depth-of-field. The selected calibration image used in the experiments corresponded to the minimum of the pattern radius. However, this procedure does not eliminate the uncertainty in the measurement. It is possible that the laser shifted within the imaging system's depth of field, that the focus on the imaging system objective lens changed slightly, or that the wavelength of light used illuminate the target pattern was disparate from the wavelength of the FLEET signal, all of which could change the perceived focus. Thus, the uncertainty caused by this variation in magnification must be considered in all velocity measurements.

\section{Camera system tilt}

There are numerous scenarios in which the camera or calibration system might be misplaced in such a way as to affect the velocity measurements. The most generic of these scenarios would be that the camera and calibration target were placed at angles relative to the primary axes of the tunnel as well as each other. Thus, not only would the optical calibration be incorrect, but the measurement would no longer be aligned with the axes of the facility. A more likely scenario would be that either the camera or the calibration target were placed at a small angle relative to the tunnel or each other. The result would be an artificial acceleration or deceleration in the flow due to the variation in the magnification along the depth-of-field. While this is certainly possible, it is very unlikely that this situation occurred in these experiments. The imaged calibration targets showed no variations in the target pattern size within individual images, which indicates that the camera and the target were aligned with each other during experimentation. Thus, the final situation to consider is one in which the camera and target were aligned with each other, but they weren't aligned with the tunnel axes. This scenario will be examined in detail.

Consider first a rotation about the $y$-axis $(x-Z$ plane). There exists a total in-plane velocity of $u_{T_{x Z}}$, where the $x$ and $z$ components are separated by an angle $\alpha$. The camera and calibration target are viewing this velocity at angle $\theta$, which is defined such that $\theta=0$ corresponds to orthogonal viewing. This scenario is shown schematically in Fig. A.2. The measured stream-wise $(x)$ velocity, $u_{x, m}$ is defined as:

while the actual stream-wise velocity $u_{x}$ is defined as:

$$
u_{x, m}=u_{T_{x z}} \cos (\alpha-\theta)
$$

$$
u_{x}=u_{T_{x z}} \cos \alpha
$$

Thus, an error $\left(\varepsilon u_{x}\right)$ can be defined between the measured stream-wise velocity and the actual:

$$
\varepsilon u_{x}=u_{x}-u_{x, m}=u_{x}\left(1-\frac{\cos (\alpha-\theta)}{\cos (\alpha)}\right)
$$

American Institute of Aeronautics and Astronautics 
In this analysis, the angle $\alpha$ is unknown and a function of the span-wise $(z)$ velocity. The angle $\theta$ is also unknown, but it can be physically bound by observation. In this situation, $\theta$ was given a normal distribution with a standard deviation equal to $1.79^{\circ}$, which corresponds to the maximum angle the target pattern could sit at relative to the tunnel axes without being observable. By evaluating this error quantity over a range of different $u_{z}$ values and the distribution of possible $\theta$ values (essentially a Monte Carlo simulation of the error), a distribution of possible values of the error were found, and the uncertainty, $\delta u_{x, x z}$, was assumed to be twice the standard deviation in the velocity distribution given by the error calculations. The above procedure was repeated for the $y-z$ plane as well (rotation about the $x$-axis), so the analysis won't be repeated here. The results of these studies are shown in Figs. 8a and 8b.

The final consideration is that of the tilt of the camera system about the $z$-axis or $x-y$ plane. This analysis is more complicated since all of the quantities were measured, and no Monte Carlo

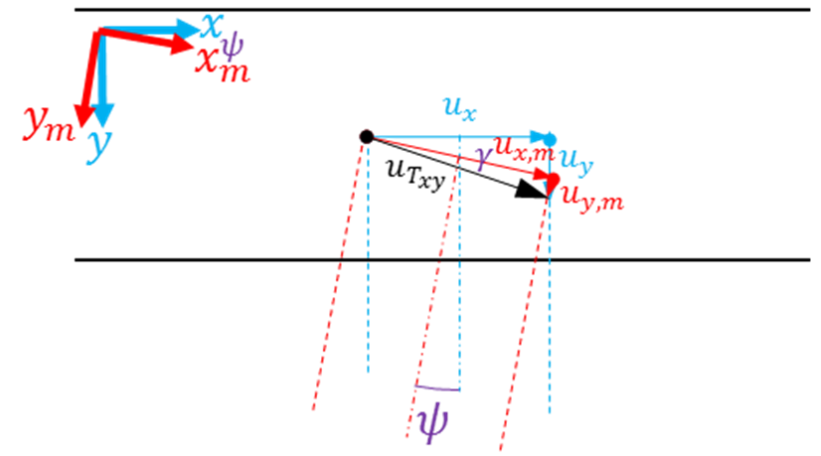

Figure A.3. Vector diagram used in the calculation of uncertainties for rotations about the $z$-axis ( $x$-y plane).

simulations were required. Consider a similar scenario to above, except that there is a total velocity $u_{T_{x y}}$, and the $x$ and $y$-components of velocity are separated by an angle $\gamma$. The camera is tilted about the $z$-axis by an angle $\psi$. This scenario is depicted in Fig. A.3. Four quantities are defined:

$$
\begin{gathered}
u_{x}=u_{T_{x y}} \cos \gamma \\
u_{x, m}=u_{T_{x y}} \cos (\gamma-\psi) \\
u_{y}=u_{T_{x y}} \sin \gamma \\
u_{y, m}=u_{T_{x y}} \sin (\gamma-\psi)
\end{gathered}
$$

Here the subscript $m$ denotes the measured quantity as above. The uncertainties in the true velocities can be expressed as (by standard Taylor expansion):

$$
\begin{aligned}
& \delta u_{x, x y}=\left[\left(\delta u_{T_{x y}} \cos \gamma\right)^{2}+\left(u_{T_{x y}} \sin \gamma \delta \gamma\right)^{2}\right]^{1 / 2} \\
& \delta u_{y, x y}=\left[\left(\delta u_{T_{x y}} \sin \gamma\right)^{2}+\left(u_{T_{x y}} \cos \gamma \delta \gamma\right)^{2}\right]^{1 / 2}
\end{aligned}
$$

In this case, the angle that is measured through the velocity calculations is $\gamma-\psi$. To evaluate the uncertainty in gamma, it is assumed that $\gamma=(\gamma-\psi)+\psi$. Since $\psi$ is unknown, it is assumed to be 0 , but it has an uncertainty again evaluated through experimental observation, which is $1.36^{\circ}$ based on the positioning of the target pattern. The uncertainty in $\gamma$ is then given by:

The angle $\gamma-\psi$ is given by:

$$
\delta \gamma=\left[(\delta(\gamma-\psi))^{2}+(\delta \psi)^{2}\right]^{1 / 2}
$$

and the uncertainty by:

$$
(\gamma-\psi)=\tan ^{-1}\left(\frac{u_{y, m}}{u_{x, m}}\right)
$$

$$
\delta(\gamma-\psi)=\frac{1}{1+\left(\frac{u_{y, m}}{u_{x, m}}\right)^{2}}\left[\left(\frac{u_{y, m}}{u_{x, m}^{2}} \delta u_{x, m}\right)^{2}+\left(\frac{1}{u_{x, m}} \delta u_{y, m}\right)^{2}\right]^{1 / 2}
$$

The uncertainties in the measured velocities are taken from the uncertainties calculated through the measurement procedure to this point. The results of all the uncertainty calculations described in this section are presented in Figs. $9 \mathrm{a}$ and $9 \mathrm{~b}$.

American Institute of Aeronautics and Astronautics 


\section{B. Analytical Uncertainties}

\section{Cross-correlation precision}

The adaptive cross-correlation procedure is described in Section III.A above. To assess the precision of the algorithm, Monte Carlo simulations were done by generating synthetic signal traces and using the algorithm on them. It was found that the maximum uncertainty of this solver within the allowable range of $R^{2}(0.95$ or greater) values was $0.1 \mathrm{px}$ or approximately $12 \mu \mathrm{m}$, though it was often better than this at higher $\mathrm{R}^{2}$ values. Nonetheless this value was used to serve as a conservative estimate of the uncertainty caused by this evaluation procedure.

\section{Velocity evaluation uncertainty}

The uncertainty imparted by the velocity evaluation is dependent on the method used. For the point-to-point method, the velocity uncertainty is given by:

$$
\delta u_{i}=\left[\left(\frac{\delta \Delta s_{i}}{\Delta t}\right)^{2}+\left(\frac{\Delta s_{i}}{\Delta t^{2}} \delta \Delta t\right)^{2}\right]^{1 / 2}
$$

Here the uncertainty in the displacement $\delta \Delta s_{i}$ is taken from the uncertainty of the cross-correlation process, and the uncertainty in the time delay, $\delta \Delta t$, is assumed to be $40 \mathrm{~ns}$ and is taken from the various uncertainties in the experimental equipment. Ultimately the uncertainty imparted by the time delay is negligible compared to that of the displacement. Although the uncertainty in the displacement are evaluated on a burst by burst and frame by frame basis, the uncertainty using the baseline value of $12 \mu \mathrm{m}$ is approximately $3 \mathrm{~m} / \mathrm{s}$ at the shortest time delays.

For the linear and polynomial fitting methods, the uncertainties require a more involved analysis. This will be demonstrated for the linear fitting method. Following the derivation in Ref. 21, the model equation is given by:

$$
s_{i}(t)=u_{i} t+s_{i, 0}
$$

Here, $s_{i}$ refers to the displacement in component $i, u_{i}$ is the fit velocity corresponding to that same directional component, and $s_{i, 0}$ is the initial position. The uncertainty in the fit parameter $u_{i}$ is derived through a series of different steps. First, a merit function is defined which determines the quality of the fit, which in this circumstance is the $\chi^{2}$ parameter:

$$
\chi^{2}\left(u_{i}, s_{i, 0}\right)=\sum_{j=1}^{n}\left(\frac{s_{i, j}-s_{i, 0}-u_{i} t_{j}}{\delta s_{i, j}}\right)^{2}
$$

where the index $j$ refers to the position within an imaging burst (frame). From this definition, the parameters $u_{i}$ and $s_{i, 0}$ are found by minimizing the $\chi^{2}$ function with respect to each fit parameter. This calculation is done by setting each of the partial derivatives equal to zero:

$$
\begin{gathered}
\frac{\partial \chi^{2}}{\partial u_{i}}=-2 \sum_{j=1}^{n} \frac{s_{i, j}-s_{i, 0}-u_{i} t_{j}}{\delta s_{i, j}^{2}}=0 \\
\frac{\partial \chi^{2}}{\partial s_{i, 0}}=-2 \sum_{j=1}^{n} \frac{t_{j}\left(s_{i, j}-s_{i, 0}-u_{i} t_{j}\right)}{\delta s_{i, j}^{2}}=0
\end{gathered}
$$

In solving these equations, several terms can be defined that simplify the process:

$$
\begin{aligned}
S & =\sum_{j=1}^{n} \frac{1}{\delta s_{i, j}^{2}} \\
S_{t} & =\sum_{j=1}^{n} \frac{t_{j}}{\delta s_{i, j}^{2}} \\
S_{s} & =\sum_{j=1}^{n} \frac{s_{i, j}}{\delta s_{i, j}^{2}} \\
S_{t t} & =\sum_{1}^{n} \frac{t_{j}^{2}}{\delta s_{i, j}^{2}} \\
S_{s t} & =\sum_{j=1}^{n} \frac{s_{i, j} t_{j}}{\delta s_{i, j}^{2}}
\end{aligned}
$$

With these terms defined, Eqs. A.4c and A.4d are reduced by substitution:

American Institute of Aeronautics and Astronautics 


$$
\begin{gathered}
s_{i, 0} S+u_{i} S_{t}=S_{s} \\
s_{i, 0} S_{t}+u_{i} S_{t t}=S_{s t}
\end{gathered}
$$

The system of equations defined by Eqs. A.4j and A.4k can be solved simultaneously to yield the fit velocity:

$$
\begin{aligned}
\Delta & =S S_{t t}-S_{t}^{2} \\
s_{i, 0} & =\frac{S_{t t} S_{s}-S_{t} S_{s t}}{\Delta} \\
u_{i} & =\frac{S S_{s t}-S_{t} S_{s}}{\Delta}
\end{aligned}
$$

To estimate the uncertainty in the velocity, the overall variance caused by the uncertainties at each point considered $\left(\delta s_{i, j}\right)$ must be measured. Following the derivation in Ref. 21, this variance can be defined as:

$$
\delta u_{i}^{2}=\sum_{j=1}^{n} \frac{\partial u_{i}}{\partial s_{i, j}}=\sum_{j=1}^{n} \frac{\left(S t_{j}-S_{t}\right)}{\delta s_{i, j}^{2} \Delta}=\frac{S}{\Delta}
$$

Finally, the uncertainty in the fit parameter $u_{i}$ is given by:

$$
\delta u_{i}=\left(\frac{S}{\Delta}\right)^{1 / 2}
$$

Procedurally, the uncertainty in the $s_{i, j}$ are determined shot by shot depending on the fit $\mathrm{R}^{2}$ value. For the baseline uncertainty of $12 \mu \mathrm{m}$, the uncertainty in the fitting procedure is found to be $0.64 \mathrm{~m} / \mathrm{s}$. The derivation for the polynomial method can be found in Ref. 21 following this same basic procedure.

\section{Appendix B. Construction of Pseudo-Streamlines}

The pseudo-streamlines presented in Fig. 17c were constructed through a series of steps that utilized all of the position data within each sampled velocity profile. Note that they are termed pseudo-streamlines in this context because the flow is intrinsically three-dimensional in character, and the construction of the streamlines utilized only 2 components of velocity within a given measurement plane. Thus, out-of-plane motion is not accounted for in this process, and the resulting streamlines are not truly streamlines by the strictest definition. Procedurally, the construction of the pseudo-streamlines was initiated during the construction of single velocity profiles. At each sampling location within the velocity profile, the average displacement between each of the 4 successive frames were calculated ( 3 displacements, 4 positions). The sample locations are shown in Fig. B.1a. Note that since the velocities were not uniform throughout the profiles, sampling locations are not uniformly distributed. For the first 3 positions in each trajectory, the local velocity was calculated using the point-to-point method described in Section III.D.1. The velocity vectors calculated through this process are shown in Fig. B.1b. The stream-wise and transverse velocities were then mapped onto a uniform mesh via cubic spline interpolation. All points falling within regions where no data were sampled were omitted from this process to avoid biasing across the transverse extent of the airfoil. Velocity vectors resulting from this calculation are shown in Fig. B.1c. Finally, the velocity fields on the uniform mesh were used to generate the pseudo-streamlines using the built-in streamslice function in MATLAB. The resulting pseudo-streamlines are repeated here for convenience in Fig. B.1d.

\section{Acknowledgments}

The authors would like to thank all of the expert staff at the NASA Langley 0.3-m TCT including Wes Goodman, Michael Chambers, Karl Maddox, Cliff Obara, Chris Cramer, Reggie Brown, Gary Beachem, and Lynn Bobbitt. These test would not have been possible without the long hours and hard work everyone contributed. Additional thanks is given to Lonnie Brown and Doug Webber for their input in the design and assessment of the test model and to Joe Morrison, Greg Jones, and Bill Milholen from NASA LaRC for their helpful discussions in choosing the test geometry. Finally, the authors would like to thank Mark Kulick for his role in fabricating the window and model mounting system and other mechanical equipment necessary for these tests. This work was funded by NASA Langley's IRAD program.

American Institute of Aeronautics and Astronautics 
a)

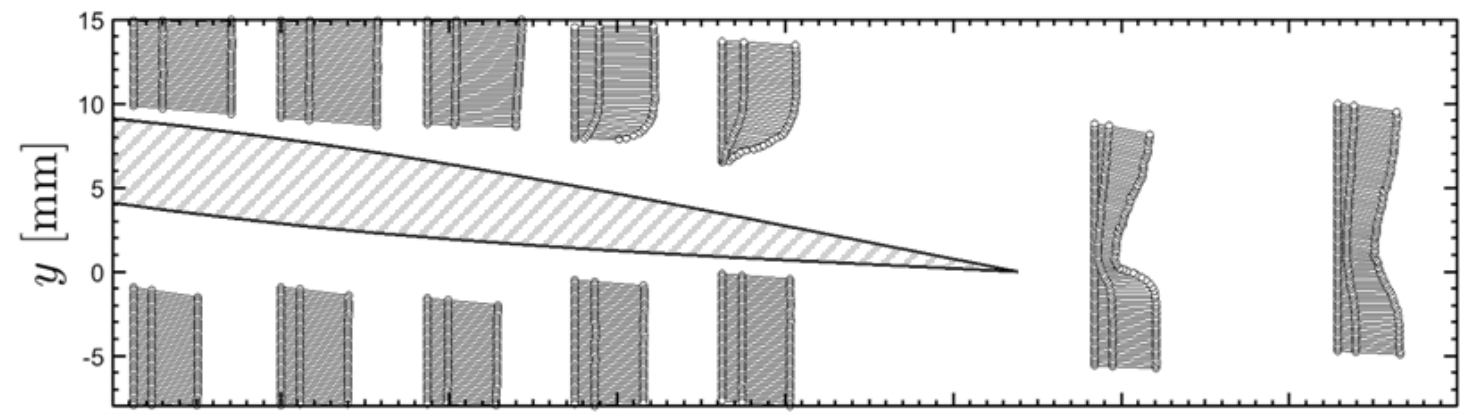

b)

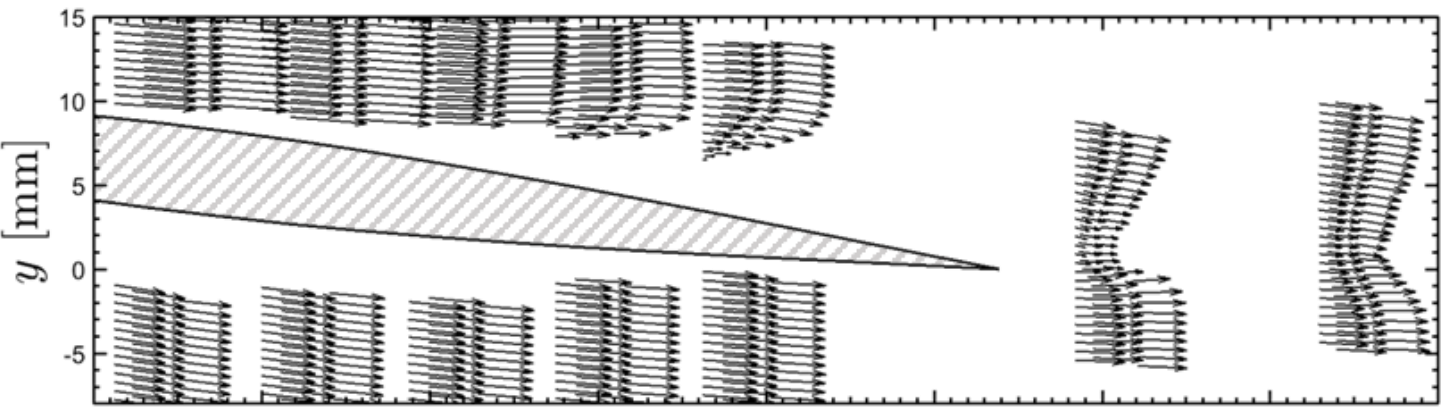

c)

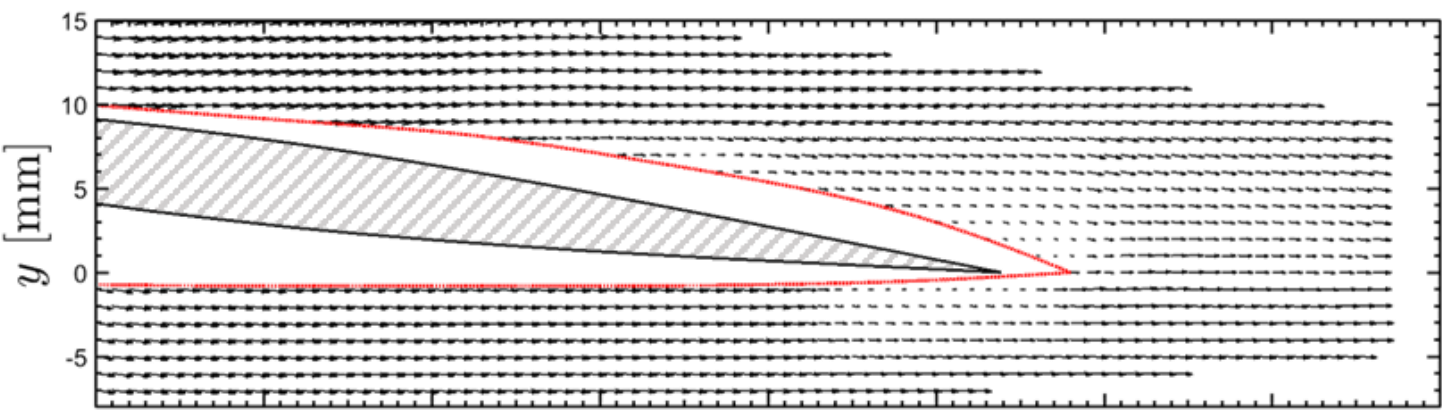

d)

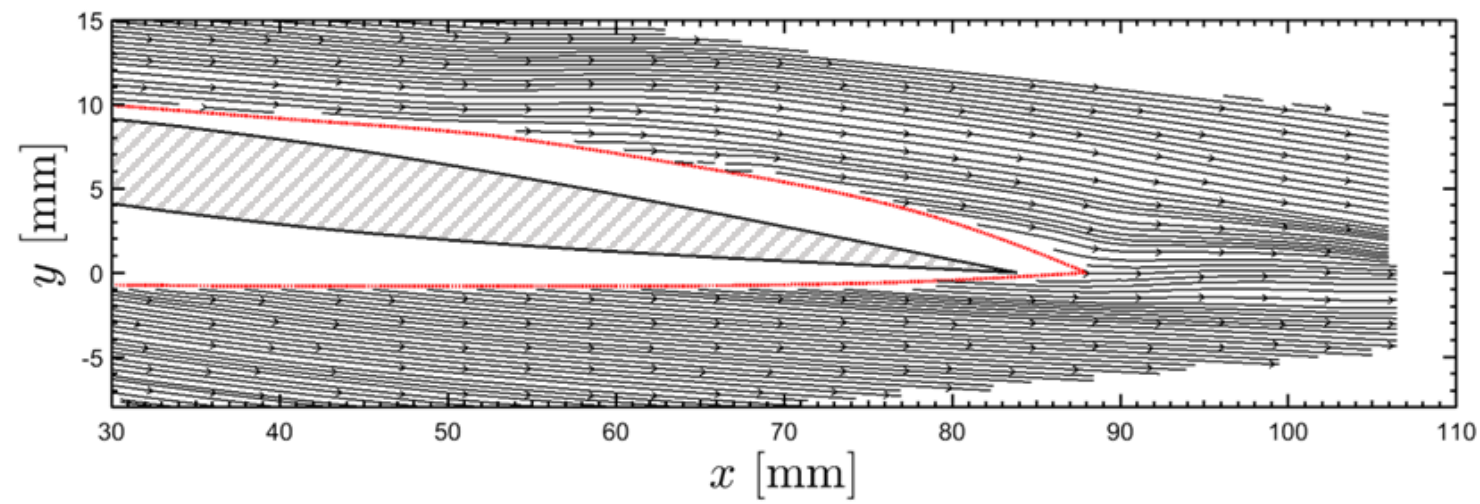

Figure B.1. Steps in the calculation of pseudo-streamlines. a) Data sampling locations, b) primary velocity vectors, $c$ ) intermediate velocity vectors, and d) final streamlines based on intermediate velocity vectors. 


\section{References}

${ }^{1}$ Ladson, C. L. and Ray, E. J. "Evolution, Calibration, and Operational Characteristics of the Two-Dimensional Test Section of the Langley 0.3-Meter Transonic Cryogenic Tunnel”, NASA TP-2749, 1987.

2 Snow, W. L., Burner, A. W., and Goad, W. K., "Image Degradation in Langley 0.3-Meter Transonic Cryogenic Tunnel," NASA Technical Memorandum 84550, 1982.

${ }^{3}$ Snow, W. L., Burner, A. W., and Goad, W. K., "Improvement in the Quality of Flow Visualization in the Langley 0.3-Meter Transonic Cryogenic Tunnel," NASA TM-87730, 1987.

${ }^{4}$ Batill, S.M., "Experimental Uncertainty and Drag Measurements in the National Transonic Facility," NASA CR $4600,1994$.

5 Fey, U., Konrath, R., Kirmse, T., Ahlefeldt, T., Kompenhans, J., and Egami, Y., "Advanced Measurement Techniques for High Reynolds Number Testing in Cryogenic Wind Tunnels," 48th AIAA Aerospace Sciences Meeting Including the New Horizons Forum and Aerospace Exposition, Orlando, FL, 2010, pp. 1-8. AIAA-2010-1301.

${ }^{6}$ Watkins, A.N., Leighty, B.D., Lipford, W.E., Oglesby, D.M., Goodman, K.Z., Goad, W.K., Goad, L.R., Massey, EA., "The Development and Implementation of a Cryogenic Pressure Sensitive Paint System in the National Transonic Facility," 47th AIAA Aerospace Sciences Meeting including The New Horizons Forum and Aerospace Exposition," Orlando, FL, 2009, pp. 1-10, AIAA-2009-421.

${ }^{7}$ Fey, U., Engler, R. H., Egami, Y., Iijima, Y., Asai, K., Jansen, U., and Quest, J. "Transition Detection by Temperature Sensitive Paint at Cryogenic Temperatures in the European Transonic Windtunnel (ETW)," 20th Internatinal Congress on Instrumentation in Aerospace Simulatin Facilities (ICIASF), Goettingen, Germany, 2003.

8 Asai, K., Amao, Y., Iijima, Y., Okura, I., and Nishide, H., "Novel Pressure-Sensitive Paint for Cryogenic and Unsteady Wind-Tunnel Testing," Journal of Thermophysics and Heat Transfer, Vol. 16, No. 1, 2002, pp. 109-115.

${ }^{9}$ Quest, J. and Konrath, R. "Accepting a Challenge - The Development of PIV for Application in pressurized cryogenic Wind Tunnels," 41st AIAA Fluid Dynamics Conference and Exhibit, Honolulu, HI, 2011.

${ }^{10}$ Willert, C., Stockhausen, G., Beversdorff, M., Klinner, J., Lempereur, C., Barricau, P., Quest, J., and Jansen, U., "Application of Doppler global velocimetry in cryogenic wind tunnels," Experiments in Fluids, Vol. 39, 2005, pp. 420-430.

${ }^{11}$ Gartrell, L. R., Gooderum, P. B., Hunter, W. W., and Meyers, J. F., "Laser Velocimetry Technique Applied to the Langley 0.3-Meter Transonic Cryogenic Tunnel," NASA TM- 81913, 1981.

${ }^{12}$ Honaker, W. C. and Lawing, P. L., "Measurements in the Flow Field of a Cylinder with a Laser Transit Anemometer and a Drag Rake in the Langley 0.3-m Transonic Cryogenic Tunnel," NASA TM- 86399, 1985.

${ }^{13}$ Michael, J. B., Edwards, M. R., Dogariu, A., and Miles, R. B., "Femtosecond laser electronic excitation tagging for quantitative velocity imaging in air," Applied Optics, Vol. 50, No. 26, 2011, pp. 5158-5162.

14 Jiang, N., Slipchenko, M.N., Mance, J.G., Felver, J., Stauffer, H., Yi, T., Danehy, P.M., and Roy, S., "Seedless Velocimetry at $100-\mathrm{kHz}$ with Picosecond Laser Electronic Excitation Tagging (PLEET)," Optics Letters. In preparation.

15 Jiang, N., Halls, B.R., Stauffer, H.U., Danehy, P.M., Gord, J.R., and Roy, S., "Selective two-photon absorptive resonance femtosecond-laser electronic-excitation tagging velocimetry," Optics Letters, Vol. 41, No. 10, 2016, pp. 2225-2228.

16 Limbach, C.M., and Miles, R.B., "Rayleigh Scattering Measurements of Heating and Gas Perturbations Accompanying Femtosecond Laser Tagging," AIAA Journal, Pre-Print, 2016.

${ }^{17}$ Burns, R.A., Danehy, P.M., Halls, B.R., and Jiang, N., "Application of FLEET Velocimetry in the NASA Langley 0.3-Meter Transonic Cryogenic Tunnel," $31^{\text {st }}$ AIAA Aerodynamic Measurement Technology and Ground Testing Conference, Dallas, TX, 2015, AIAA-2015-2566.

${ }^{18}$ Burns, R.A., Peters, C.J., and Danehy, P.M., "Femtosecond-Laser-Based Measurements of Velocity and Density in the NASA Langley 0.3-m Transonic Cryogenic Tunnel," 32 ${ }^{\text {nd }}$ AIAA Aerodynamic Measurement Technology and Ground Testing Conference, Washington, D.C., 2016.

${ }^{19}$ Burns, R.A., Danehy, P.M., Halls, B.R., and Jiang, N., "Unseeded Velocimetry in a Transonic, Cryogenic Wind Tunnel Using FLEET," AIAA Journal. Accepted

${ }^{20}$ Peters, C.J., Miles, R.B., Burns, R.A., Danehy, P.M., Bathel, B.F., and Jones, G.S., "Femtosecond Laser Tagging Characterization of a Sweeping Jet Actuator Operating in the Compressible Regime", 32 ${ }^{\text {nd }}$ AIAA Aerodynamic Measurement Technology and Ground Testing Conference, Washington, D.C., 2016.

${ }^{21}$ Press, W.H., Flannery, B.P., Teukolsky, S.A., and Vetterling, W.T., Numerical Recipes in C: The Art of Scientific Computing. Cambridge University Press, New York, 1988, Chap. 14.

American Institute of Aeronautics and Astronautics 